\title{
Feature-Based Groupwise Registration by Hierarchical Anatomical Correspondence Detection
}

\author{
Guorong Wu,' Qian Wang, ${ }^{1,2}$ Hongjun Jia,' and Dinggang Shen ${ }^{1 *}$ \\ ${ }^{1}$ Department of Radiology and BRIC, University of North Carolina at Chapel Hill, North Carolina \\ ${ }^{2}$ Department of Computer Science, University of North Carolina at Chapel Hill, North Carolina
}

\begin{abstract}
Groupwise registration has been widely investigated in recent years due to its importance in analyzing population data in many clinical applications. To our best knowledge, most of the groupwise registration algorithms only utilize the intensity information. However, it is well known that using intensity only is not sufficient to achieve the anatomically sound correspondences in medical image registration. In this article, we propose a novel feature-based groupwise registration algorithm to establish the anatomical correspondence across subjects by using the attribute vector that is defined as the morphological signature for each voxel. Similar to most of the state-of-the-art groupwise registration algorithms, which simultaneously estimate the transformation fields for all subjects, we develop an energy function to minimize the intersubject discrepancies on anatomical structures and drive all subjects toward the hidden common space. To make the algorithm efficient and robust, we decouple the complex groupwise registration problem into two easy-to-solve subproblems, namely (1) robust correspondence detection and (2) dense transformation field estimation, which are systematically integrated into a unified framework. To achieve the robust correspondences in the step (1), several strategies are adopted. First, the procedure of feature matching is evaluated within a neighborhood, rather than only on a single voxel. Second, the driving voxels with distinctive image features are designed to drive the transformations of other nondriving voxels. Third, we take advantage of soft correspondence assignment not only in the spatial domain but also across the population of subjects. Specifically, multiple correspondences are allowed to alleviate the ambiguity in establishing correspondences w.r.t. a particular subject and also the contributions from different subjects are dynamically controlled throughout the registration. Eventually in the step (2), based on the correspondences established for the driving voxels, thin-plate spline is used to propagate correspondences on the driving voxels to other locations in the image. By iteratively repeating correspondence detection and dense deformation estimation, all the subjects will be aligned onto the common space. Our feature-based groupwise registration algorithm has been extensively evaluated over 18 elderly brains, 16 brains from NIREP (with 32 manually delineated labels), 40 brains from LONI LPBA40 (with 54 manually delineated labels), and 12 pairs of normal controls and simulated atrophic brain images. In all experiments, our algorithm achieves more robust and accurate registration results, compared with another groupwise algorithm and a pairwise registration method. Hum Brain Mapp 33:253-271, 2012. @ 2011 Wiley Periodicals, Inc.
\end{abstract}

Key words: groupwise registration; feature matching; attribute vector; hierarchical deformable registration; thin-plate spline

${ }^{*}$ Correspondence to: Dinggang Shen, Department of Radiology and BRIC, University of North Carolina at Chapel Hill, NC 27599, U.S.A. E-mail: dgshen@med.unc.edu

(C) 2011 Wiley Periodicals, Inc.
Received for publication 19 April 2010; Revised 18 August 2010; Accepted 20 October 2010

DOI: $10.1002 / \mathrm{hbm} .21209$

Published online 9 March 2011 in Wiley Online Library (wileyonlinelibrary.com). 


\section{INTRODUCTION}

Registration of image population has received more and more attentions in recent years due to its importance in analyzing population data [Crum et al., 2004; Maintz and Viergever, 1998; Zitová and Flusser, 2003]. However, selection of any individual subject as a template will eventually lead to bias in registering all subjects, as pointed in [Allassonnière et al., 2007; Blezek and Miller, 2007; Sabuncu et al., 2009]. To overcome this problem, groupwise registration has been proposed and become popular recently, because it is able to register all images in the population simultaneously and also find the population center at the same time.

Specifically, groupwise registration method can register all images without selection of an explicit template (which is generally required by the pairwise registration algorithms), thus it is attractive to the construction of unbiased atlas and the precise analysis of population data. However, this also makes groupwise registration much more complicated than the pairwise registration. Although groupwise registration can be obtained by exhausting pairwise registrations between all possible subject pairs in the population, it suffers from very heavy computation. For example, in Seghers' method [Seghers et al., 2004], each individual subject is selected as the template in roundrobin fashion and registered with all other subjects independently. According to their article [Seghers et al., 2004], it takes about 170 days on one $2.6-\mathrm{GHz}$ processor to perform 4,032 pairwise registrations for a group of 64 subjects.

More favorable approaches are later proposed to align all subjects simultaneously, following the groupwise concept explicitly. For example, Joshi et al. [2004] iteratively register the subjects to the group mean image and construct the atlas (group mean) as the Fréchet mean of all registered images. They have further extended their work to longitudinal analysis of the human brains aged from 22 to 79 years old by kernel regression [Davis et al., 2007]. One limitation of this method is that the generated mean image is very fuzzy initially, because deformations cannot be accurately predicted in the very beginning of registration. This limitation can, therefore, undermine the performance of the algorithm by simply forcing the subjects (with clear anatomical structures) to align with the fuzzy mean image, where the detailed structural information is lost through simple image averaging. Similar work can be found in [Geng et al., 2009], which estimates the correspondences among a group of images by jointly registering them to an implicit reference corresponding to the group mean image.

The congealing method has been proposed in [LearnedMiller, 2006; Miller et al., 2000] to jointly warp the subjects toward a hidden common space by minimizing the sum of stack entropies in the population. Balci et al. [2007] further implement the congealing method for nonrigid registration of brain images by modeling transformations with B-Splines. However, the method is intensity-based and, thus, intrinsically insufficient to establish good anatomical correspondences across subject images during the registration [Shen and Davatzikos, 2002]. By formulating groupwise registration as numerical optimization, Balci's algorithm uses the steepest descent method for solving groupwise registration, which is unfortunately sensitive to local minima. Also, the cost function is estimated based on only $\sim 1 \%$ randomly sampled voxels, regardless of their morphological importance in the image domain, which can also affect the registration performance.

On the other hand, diffeomorphism has become popular in the field of computational anatomy, to mathematically describe the warping trajectory of minimal energy in the Riemannian manifolds, instead of the Euclidian space [Avants et al., 2008; Beg et al., 2005; Cootes et al., 2004; Joshi et al., 2004; Vercauteren et al., 2009b]. Its "one-toone" and "onto" properties assure a smooth transformation field with no folding. Based on the diffeomorphic metric, Marsland et al. [2008] formulate groupwise registration as a learning based approach, where point correspondences are solved using a minimum description length objective function. Accordingly, they can obtain reasonable alignment results with the minimal length in encoding a set of images and the corresponding transformation fields.

In summary, many groupwise registration methods have been proposed; however, most of them are intensitybased, and all voxels in the image domain are equally treated [Balci et al., 2007; Joshi et al., 2004] regardless of their morphological importance. None of the articles above addresses the issue of anatomical correspondence, which is critical to the accurate registration and comparison of images. To overcome these limitations, we propose a novel feature-based groupwise registration framework, which uses attribute vector as the signature of each voxel to identify correspondences across different images in the population. As the selection of image features is not the main focus of this article, we simply adopt the features used in [Shen and Davatzikos, 2002] as attributes for guiding groupwise registration, due to their good performance in pairwise registration. Specifically, the features of each voxel include not only the image intensity but also edge information and geometric moment invariants (GMIs) calculated from tissue maps of white matter (WM), gray matter (GM), and ventricular cerebrospinal fluid (CSF). It is worth noting that other features, such as those calculated from local histograms [Shen, 2007], SaS filters [Liao and Chung, 2008], and the optimal feature combination [Wu et al., 2006b], can also be used and incorporated into our groupwise registration framework.

Any registration problem, regardless of pairwise or groupwise, is eventually solved by an optimization approach. Most contemporary optimization methods [Balci et al., 2007; Christensen, 1999; Rueckert et al., 1999; 
Thirion, 1998; Vercauteren et al., 2009a] use the gradientbased approach to minimize a certain cost function by moving each voxel along its specific direction. These optimization methods are straightforward in estimating the transformation fields. However, they are always challenged by local minima, which are prevalent especially when concerning the neuroanatomical complexity. Moreover, the resulting transformation fields are often "bumpy" due to independent warping voxels in their neighborhoods. To attack these problems, we propose a hierarchical groupwise registration framework in which the groupwise registration problem is decoupled into two easy-to-solve subproblems: (1) establish reliable correspondences only on a small number of the driving voxels by feature matching and (2) estimate dense transformation fields according to the sparse correspondences established on the driving voxels.

The quality of sparse correspondences established in the first subproblem is critical to our feature-based groupwise registration algorithm. To this end, we first estimate correspondences only for the driving voxels with distinctive attribute vectors, that is, those located at sulcal roots, gyral crowns, and ventricular corners [Shen and Davatzikos, 2002; Wu et al., 2006a, 2007], because it is relatively easy to detect their correspondences. The resulted sparse correspondences on the driving voxels can be used to steer the deformations of the rest voxels. After brain structures have been roughly aligned based on the correspondences of the initial driving voxels, more voxels (with less distinctive attributes) can be considered as driving voxels to further refine the registration result. Moreover, the robustness of our registration algorithm is further improved by the following three ways: (1) Correspondence is evaluated by considering all candidates in the search neighborhood, using not only the voxelwise but also the regionwise similarities to measure the likelihood of the corresponding voxels; (2) Soft correspondences (or multiple correspondences) in the spatial domain are allowed initially [Chui and Rangarajan, 2003; Shen, 2009] and gradually discouraged with progress of registration to establish the exact one-to-one correspondence in the end of registration; (3) Similar soft assignment is also applied to the subjects in the population, that is, all subjects are allowed to guide the alignment of a particular subject in the beginning of registration, while only the most similar subjects are allowed in the end of registration.

In the second subproblem, we use the thin-plate spline (TPS) [Bookstein, 1989b; Chui and Rangarajan, 2003; Wu et al., 2010] to propagate sparse correspondences (on the driving voxels) to the whole image domain for producing the dense transformation fields and warping all brain images immediately. The TPS parameters, which consist of affine and deformable counterparts, are computed by considering the driving voxels as control points. The deformation of each nondriving voxel can then be interpolated based on the control points.
The performance of our groupwise registration algorithm has been compared with the congealing method [Balci et al., 2007] and the pairwise HAMMER registration algorithm [Shen and Davatzikos, 2002, 2003], using various real and simulated datasets. The real datasets include 18 elderly brain subjects [Resnick et al., 2000], 16 NIREP brain images [Allassonnière et al., 2007], and LONI LPBA40 dataset [Shattuck et al., 2008]. For all these three datasets, our proposed algorithm can achieve the best performance on registration accuracy and consistency (in terms of labeling ROIs and producing group mean images). The simulated dataset includes 12 pairs of normal controls and simulated atrophic brains [Xue et al., 2006]. Via paired $t$-tests on Jacobian determinants of the estimated transformation fields using the SPM software package (http://www.fil. ion.ucl.ac.uk/spm/), our proposed algorithm achieves the highest $t$-scores in detecting simulated brain atrophies from those 12 pairs of images, compared with the other two registration methods under comparison.

In the following, we will first present the framework of our groupwise registration algorithm in "The Framework of Feature-Based Groupwise Registration Method" Section. To address the importance of correspondence detection in groupwise registration, we adopt some state-of-the-art strategies to achieve the robust correspondence in "Robust Correspondence Detection" Section. After the brief summary in "Summary And Implementation Issues" Section, the extensive experiments have been performed in "Experiments" Section to demonstrate the performance of our proposed method. Finally, we will conclude and discuss our future work in "Conclusions" Section.

\section{THE FRAMEWORK OF FEATURE-BASED GROUPWISE REGISTRATION METHOD}

Given a group of subjects $S=\left\{S_{i} \mid i=1, \ldots, N\right\}$, the essential of groupwise registration is to find a set of forward transformations $G=\left\{g_{i} \mid g_{i}(x)=x+h_{i}(x), x \in \Omega_{i} \subset\right.$ $\left.\mathfrak{R}^{3}, i=1, \ldots, N\right\}$, which are defined in the subject domain $\Omega_{i}$ and can transform each subject toward the hidden common space $\Omega_{c}$ with the displacement $h_{i}(x)$. Figure 1 shows the schematic illustration of the groupwise registration. Specifically, for each subject $S_{i}$ in the group, its (dense) forward transformation field $g_{i}$ (i.e., red solid arrows) is estimated to warp the subject $S_{i}$ toward the common space (i.e., the purple circled region in the center of Fig. 1). As a result, the anatomical differences across all registered subjects will be minimized after groupwise registration. To connect each pair of subjects via the common space $\Omega_{c}$, the inverse transformation fields $G^{-1}=\left\{g_{i}^{-1} \mid g_{i}^{-1}(x)=x+\right.$ $\left.h_{i}^{-1}(x), x \in \Omega_{c} \subset \mathfrak{R}^{3}, i=1, \ldots, N\right\}$ must be calculated, as shown in Figure 1. Thus, the compositional transformation $g_{j}^{-1} \circ g_{i}$ can warp subject $S_{i}$ to $S_{j}$ and $g_{i}^{-1} \circ g_{j}$ from subject $S_{j}$ to $S_{i}$ (as demonstrated in the right panel of Fig. 1). In the following, we use $i$ to index the subject under consideration and $j$ for any other subject except $S_{i}$. Also, we call 


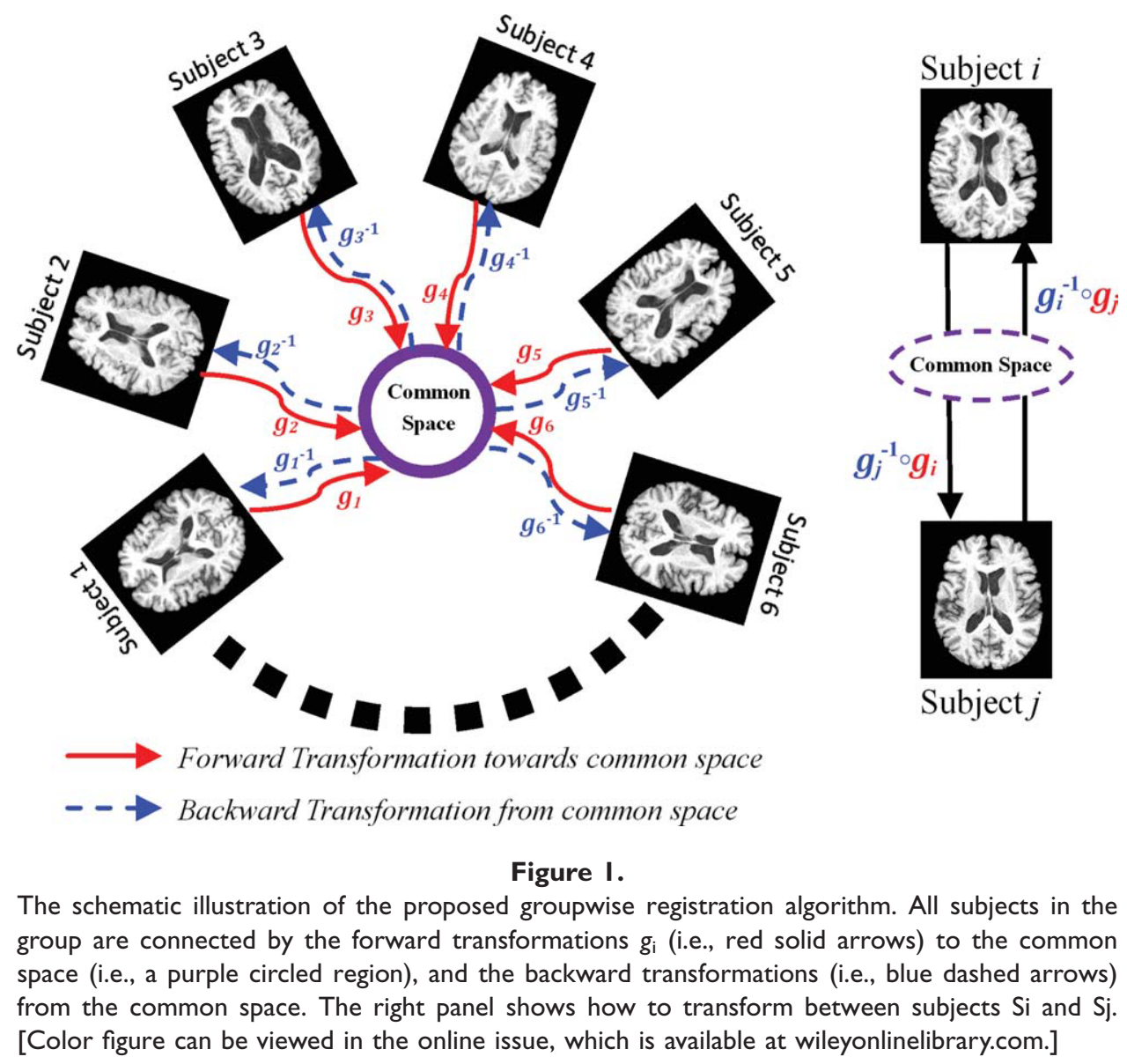

$G$ the set of forward transformations and $G^{-1}$ the set of backward transformations, which enter and leave the common space, respectively.

As a feature-based groupwise registration method, one major contribution in our work is that we use attribute vector as the morphological signature of each voxel, to achieve robust anatomical correspondence. Without loss of generality, the same attribute vector in HAMMER algorithm [Shen and Davatzikos, 2002] is used in our groupwise registration algorithm by using the brain images that have been segmented into WM, GM, and CSF. In particular, the attribute vector $\vec{a}(x)=\left[\vec{a}(x), \vec{a}^{2}(x), \vec{a}(x)\right]$ is made up of three parts: (1) a $1 \times 1$ vector representing the edge

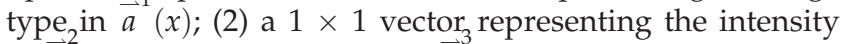
in $\vec{a}^{2}(x)$; and (3) GMIs vector $\vec{a}^{3}(x)$ [Lo and Don, 1989] of WM, GM, and CSF. The domain of the edge type has seven discrete values, namely nonedge and six possible edge types between WM, GM, and CSF. For each tissue type, GMIs are calculated in multiscales from zero-order to second-order to describe the local anatomical shape.

As shown in Figure 1, all subjects in the group will agglomerate together in the hidden common space, where the difference between each pair of subjects is minimized. Also, the estimated transformations will be constrained by a certain regularization term $\operatorname{Reg}($.$) . The overall energy$ function used in our groupwise registration can be formulated as below:

$E(G)=\sum_{i=1}^{N} \sum_{x \in \Omega_{i}}\left\{\sum_{j=1, j \neq i}^{N} D\left(\vec{a}_{i}(x), \vec{a}_{j}\left(g_{j}^{-1} \circ g_{i}(x)\right)\right)\right\}+\operatorname{Reg}(G)$,

where $D($.$) denotes the distance measurement between at-$ tribute vectors of corresponding voxels in two different subjects. The term $g_{j}^{-1} \circ g_{i}$ is used to map voxel $x$ from subject domain $\Omega_{i}$ to the domain $\Omega_{j}$ of subject $S_{j}$, through the common space $\Omega_{c}$. Note that we will extend voxelwise $D($.$) to regionwise feature-matching measurement in$ "Regionwise Feature Matching" Section, to achieve the robust anatomical correspondences across subjects.

Although many mathematically sound algorithms have been proposed to minimize the energy function, most of them, for example, the Gauss-Newton method, are computationally expensive and could be vulnerable to local minima. To cast the groupwise registration into the optimization of a well-posed problem, we approximate the energy function $E(G)$ in two ways.

First, voxels with distinctive attribute vectors, called as driving voxels, will be first selected to drive the 
registration, because they are more reliable to identify their correspondences across different subjects. These voxels are typically located at ventricular corners, sulcal roots, and gyrus crowns. By following a criterion proposed in [Shen and Davatzikos, 2002], a set of driving voxels can be hierarchically selected by adjusting a threshold on the zero-order GMIs of all voxels. Here, we denote the set of driving voxels in each subject $S_{i}$ as $\mathrm{DV}_{i}=\left\{x_{i}(m) \mid m=\right.$ $\left.1, \ldots, M_{i}\right\}$ where $M_{i}$ is the overall number of driving voxels. Therefore, we plan to determine the correspondences only for the driving voxels $\left\{x_{i}(m)\right\}$ of each subject $S_{i}$, instead of all voxels in all subjects. Other nondriving voxels will follow the transformations estimated for the driving voxels. The use of the driving voxels facilitates the hierarchical registration and also leads to (1) fast registration due to the requirement of establishing correspondences only for very few driving voxels, and (2) accurate registration due to the reliable detection of correspondences for the driving voxels with distinctive attribute vectors.

Second, we introduce a collection of correspondence fields $F=\left\{f_{i}(x) \mid x \in \Omega_{i} \subset \mathfrak{R}^{3}, i=1, \ldots, N\right\}$ in our method to simply the optimization of $E(G)$. As described next, one advantage on the introduction of $F$ is that it decouples the complex optimization problem into two relative easy-tosolve subproblems [Cachier et al., 2003], that is, (1) establishing a set of correspondences $f_{i}(x)$ by feature matching and (2) finding the transformation field $g_{i}$ to fit the corresponding field $f_{i}(x)$ and also satisfy the regularization term $\operatorname{Reg}(G)$ in Eq. (1).

By taking the advantage of the driving voxels and introduction of the correspondence field $F$, we can further define the energy function for our groupwise registration framework as below:

$$
\begin{aligned}
E(F, G)=\sum_{i=1}^{N} & \sum_{m=1}^{M_{i}}\left\{\sum_{j=1, j \neq i}^{N} D\left(\vec{a}_{i}\left(x_{i}(m)\right)\right), \vec{a}_{j}\left(g_{j}^{-1} \circ f_{i}\left(x_{i}(m)\right)\right)\right. \\
& \left.+\left\|f_{i}\left(x_{i}(m)\right)-g_{i}\left(x_{i}(m)\right)\right\|^{2}\right\}+\operatorname{Reg}(G) .
\end{aligned}
$$

It can be observed that Eq. (2) depends on the two vector fields, that is, $F$ and $G$. We propose to solve $F$ and $G$ separately by the two steps. In the first step $\left(\mathbf{S P}_{\mathbf{1}}\right)$, by fixing all previous estimated transforms $G$ and $G^{-1}$, we will estimate the new correspondence field $\hat{F}$ via correspondence detection. Then, in the second step $\left(\mathbf{S P}_{\mathbf{2}}\right)$, based on the correspondence field $\hat{F}$ estimated in the first step, we will estimate the new transformations $\hat{G}$. These two steps are repeated during the registration, with details described below.

\section{$S P_{1}$ : Estimate Sparse Correspondence Field $\boldsymbol{F}$}

Step 1 optimizes the correspondence field $F$ in the brace of Eq. (2) by fixing the forward transforms $G$ and the inverse $G^{-1}$. The energy function in this step is defined as:

$$
\begin{array}{r}
E_{1}(\hat{F})=\sum_{i=1}^{N} \sum_{m=1}^{M_{i}}\left\{\sum_{j=1, j \neq i}^{N} D\left(\vec{a}_{i}\left(x_{i}(m)\right), \vec{a}_{j}\left(g_{j}^{-1} \circ \hat{f}_{i}\left(x_{i}(m)\right)\right)\right)\right. \\
\left.+\left\|\Delta f\left(x_{i}(m)\right)\right\|^{2}\right\}
\end{array}
$$

where $\Delta f_{i}\left(x_{i}(m)\right)=\hat{f}_{i}\left(x_{i}(m)\right)-g_{i}\left(x_{i}(m)\right)$ denotes the update of correspondence on $x_{i}(m)$ from $g_{i}\left(x_{i}(m)\right)$ in the beginning to $\hat{f}_{i}\left(x_{i}(m)\right)$ in the end of each round of groupwise registration. As we only consider correspondence detection on the driving voxels, $\Delta f_{i}$ has possible nonzero value only on a small number of the driving voxels, and it is zero on all other voxels. To find the optimal correspondence $\hat{f}_{i}\left(x_{i}(m)\right)$ on each driving voxel $x_{i}(m)$, we perform a greedy search by evaluating the feature matching [in the brace of Eq. (3)] on each voxel in a search neighborhood $n_{s}$ of the driving voxel $x_{i}(m)$. Moreover, as we will point out in the next section, some special strategies are needed to deal with the difficult correspondence detection problem in the brain images. Specifically, to achieve the robust correspondence detection, we will replace the voxelwise feature matching $D(.,$.$) by a more reliable regionwise matching and will also$ use soft assignment to alleviate the image ambiguities in registration. We will make them clear in "Robust Correspondence Detection" Section.

\section{$S P_{2}$ : Estimate Dense Transformation Field $\mathbf{G}$}

By fixing $\hat{F}$, the dense transformations $\hat{G}$ can be estimated by minimizing $E_{2}$ below:

$$
\begin{gathered}
E_{2}(\hat{G})=\sum_{i=1}^{N}\left\{\sum_{m=1}^{M_{i}}|| \hat{f}_{i}\left(x_{i}(m)\right)-\hat{g}_{i}\left(x_{i}(m)\right)||^{2}\right\}+\operatorname{Reg}(\hat{G}) \\
\text { s.t. } \quad \forall x \in \Re^{3}, \sum_{i=1}^{N} \hat{h}_{i}(x)=0 .
\end{gathered}
$$

The constraint given above is used to limit the "global drift" across subjects [Bhatia et al., 2004; Studholme and Cardenas, 2004], that is, requiring the sum of all displacements $\hat{h}_{i}(x)$ at location $x$ of different subjects to vanish and, thus, ensuring that the common space be the unbiased center of the population [Balci et al., 2007]. The regularization term is defined by the bending energy in this article as follows:

$$
\operatorname{Reg}(\hat{G})=\sum_{i=1}^{N}\left\|L\left(\hat{g}_{i}\right)\right\|^{2}
$$

$$
\left\|L\left(\hat{g}_{i}\right)\right\|^{2}=\sum_{l_{1}+l_{2}+l_{3}=2} \frac{1}{l_{1} ! l_{2} ! l_{3} !} \iiint\left(\frac{\partial^{2}\left(\hat{g}_{i}\right)}{\partial x_{1}^{l_{1}} \partial x_{2}^{l_{2}} \partial x_{3}^{l_{3}}}\right)^{2} d x_{1} d x_{2} d x_{3},
$$


where $l_{i}$ is 0,1 , or 2 . $L($.) denotes an operator on each transformation field $\hat{g}_{i}$, to constrain its second-order derivatives. TPS can be used to efficiently interpolate the dense and smooth transformation field $\hat{G}$ by minimizing the bending energy of transformation fields [Eq. (5)] and preserving the correspondence detection results on $x_{i}(m)$ (i.e., $\hat{g}_{i}\left(x_{i}(m)\right)=\hat{f}_{i}\left(x_{i}(m)\right)$ ).

\section{ROBUST CORRESPONDENCE DETECTION}

As mentioned in "The Framework of Feature-Based Groupwise Registration Method" Section, robust correspondences are very important for our groupwise registration algorithm to achieve sound anatomical alignment. Given the estimated correspondence field $\hat{F}$, the estimation of the transformation field $\hat{G}$ is straightforward by using TPS interpolation as described in the second step above. Therefore, in this section, we will mainly focus on the description of our correspondence detection strategies. We will first demonstrate the importance of using both regionwise feature matching and soft assignment strategies (in "Regionwise Feature Matching, Soft Correspondence Assignment, and Fuzzy Control of Soft Assignment" Sections). Based on these, we will discuss how to further improve the energy function of $E_{1}$ in Eq. (3), to achieve more robust correspondence detection ("Improved Energy Function in SP" Section). Finally, we will present our solution to obtaining good correspondences in the groupwise registration ("Solution to the Energy Function" Section).

\section{Regionwise Feature Matching}

We have proposed to use the attribute vector, instead of only image intensity, to determine the correspondence of each driving voxel. However, it might be still not sufficient for robust correspondence detection, due to the complexity of brain structures. Regionwise feature matching is thus proposed, with its benefit in correspondence detection illustrated in Figure 2. The top row of Figure 2 shows the procedure of refining correspondence for a voxel $x_{i}$ (a red solid triangle in the top left of Fig. 2), whose ideal destination should be at the upper-right corner of the ventricle (a red star) in the common space (the top middle of Fig. 2). Assume the voxel $x_{i}$ has been warped to $f_{i}\left(x_{i}\right)$ (a red triangle in the top middle of Fig. 2) according to the previously estimated forward transformation G. Different from pairwise registration algorithm, the correspondence detection in the groupwise registration should: (1) consider all other subjects, instead of just one deterministic template image; (2) compare all pairs of subjects by concatenating the forward transformation and the backward transformation via the hidden common space. When updating the correspondence of the voxel $x_{i}$ w.r.t. a given subject $S_{j}$ (the top right of Fig. 2), several candidates in $S_{j}$ (blue dots) can be detected, with their locations $\left(v_{1} \sim v_{3} \in n_{S}\right)$ in the common space as indicated by green rectangles. All candidates could be qualified as correspondences, if only voxelwise feature matching is used. However, if we inspect their attribute vectors in the subvolume (with the enlarged views displayed in the bottom row of Fig. 2), the candidate (1) which is located exactly at the upper-right ventricular corner of $S_{j}$ will beat the other two confounding locations (2) and (3).

Based on this observation, we replace the voxelwise measurement $D($.$) by the regionwise feature-matching$ measurement. First, instead of evaluating $D($.$) in the sub-$ jects' domains [in Eqs. (1-3)], we calculate $D$ in the common space $\Omega_{c}$. As shown in Figure 2, for the driving voxel $x_{i}$ with the latest estimated correspondence $f_{i}\left(x_{i}\right)$, we will check each of its possible correspondence candidates $v$ in the search neighborhood $n_{S} \subset \Omega_{c}$ of $f_{i}\left(x_{i}\right)$ in the common space, to determine a possible update $\Delta f_{i}\left(x_{i}\right)=v-f_{i}\left(x_{i}\right)$. Once $\Delta f_{i}\left(x_{i}\right)$ is determined, all voxels $z$ in the neighborhood $n_{r} \subset \Omega_{i}$ of the voxel $x_{i}$ will be considered to compare their attribute vectors with their counterpart in subject $S_{j}$. Note that he effect of $\Delta f_{i}\left(x_{i}\right)$ on $x_{i}$ is propagated onto the neighboring voxels $z$ of $n_{r}$ at a Gaussian way, that is, $\Delta\left(x_{i}\right.$, $z)=e^{-\left(x_{i}-z\right)^{2} / \sigma^{2}}$. The correspondence of voxel $z$ can thus be located in subject $S_{j}$ by concatenating $f_{i}\left(x_{i}\right), \Delta f_{i}\left(x_{i}\right) . \Delta\left(x_{i}, z\right)$, and the backward transformation $g_{j}^{-1}(v)$ (i.e., following the red, green, and blue dash arrows in Fig. 2). Accordingly, our regionwise feature-matching measurement is defined as:

$$
\begin{aligned}
& D_{i \rightarrow j}\left(\vec{a}_{i}\left(x_{i}\right), \Delta f_{i}\left(x_{i}\right)\right) \\
& \quad=\sum_{z \in n_{r}\left(x_{i}\right)} D\left(\vec{a}_{i}(z), \vec{a}_{i}\left(g_{j}^{-1}\left[f(z)+\Delta f_{i}\left(x_{i}\right) \cdot \Delta\left(x_{i}, z\right)\right]\right)\right),
\end{aligned}
$$

where $D($.$) is the voxelwise feature-matching measurement$ used in Eq. (1).

\section{Soft Correspondence Assignment}

Besides using the regionwise feature-matching measurement as defined above in Eq. (6), we further use a soft assignment strategy to attack the ambiguities in correspondence detection. This strategy can help avoid the potential mismatches in large variable brain regions of different subjects, that is, cortical regions. Figure 3 demonstrates a difficult case typically happening in brain registration. The red circle denotes a driving voxel $x_{i} \in S_{i}$ at the crown of the precentral gyrus (PCG). In the beginning of registration, it may have several candidate correspondences in subject $S_{j}$, such as the voxels at the crowns of middle frontal gyrus (MFG), precentral gyrus (PrG), and postcentral gyrus (PoG), respectively. These candidate locations are displayed with pink solid circles in $S_{j}$ (Fig. 3b) and their same locations are also shown in $S_{i}$ by pink dashed circles (Fig. 3a,c).

As these three candidates are all located at the crown of gyrus and share similar morphological features, it would 


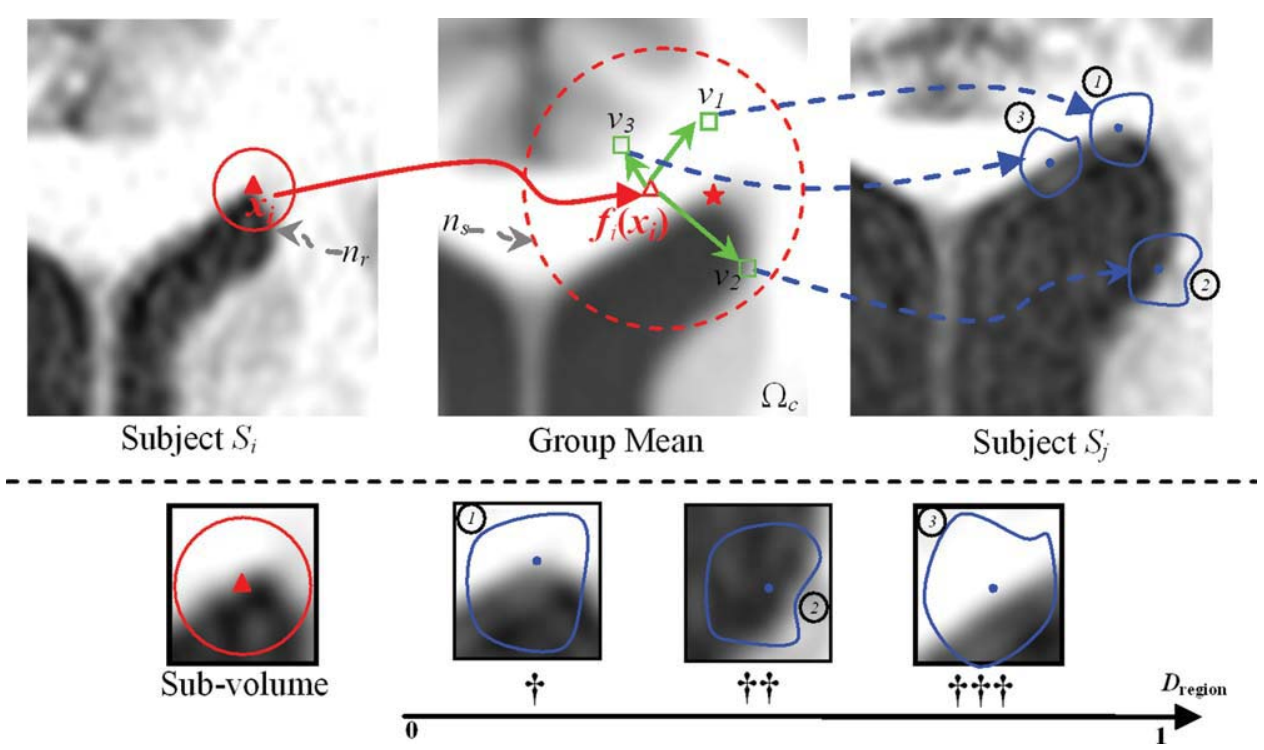

Figure 2.

Demonstration of the advantage of using the subvolume matching to measure the regionwise difference. The top row shows that the voxelwise difference is not sufficient for reliable correspondence detection since the attribute vector of voxel $x i$ is similar to three candidates (blue dots). The ambiguity by voxelwise difference can be clarified when the regionwise difference

$\mathrm{D}$ is considered (as displayed in the bottom row). Note that the blue curve denotes a neighborhood in the Subject $\mathrm{Sj}$, deformed from the original spherical neighborhood in the Subject Si. [Color figure can be viewed in the online issue, which is available at wileyonlinelibrary.com.]

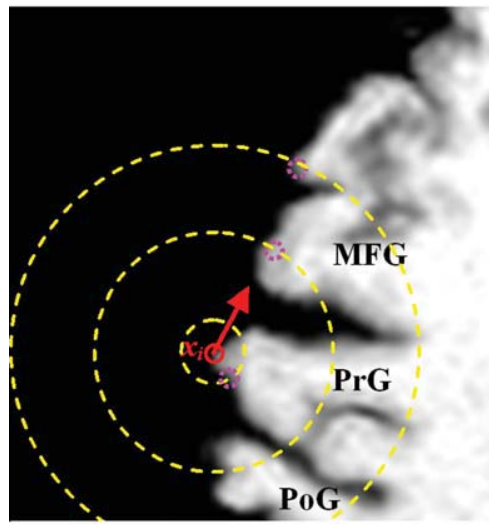

(a) Subject $S_{i}$

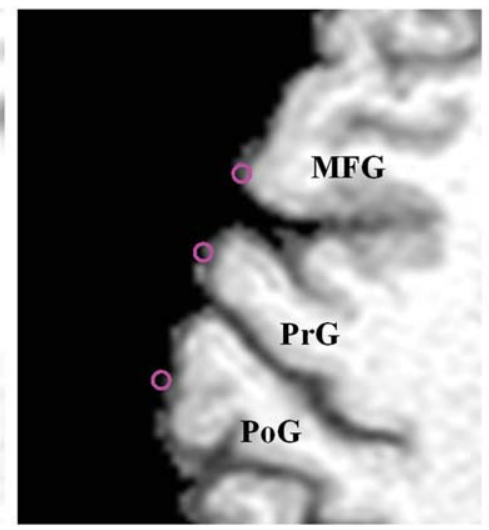

(b) Subject $S_{j}$

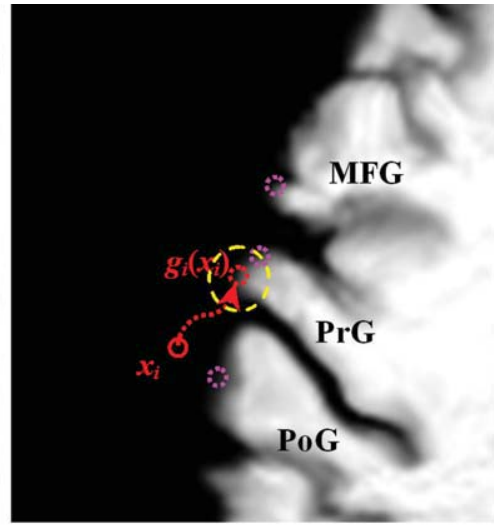

(c) Tentatively warped subject $S_{i}$

Figure 3.

Demonstration of the importance of using soft assignment for correspondence detection. The red circle denotes a voxel xi under consideration, which is located at the crown of PrG of Si. In determining the correspondence w.r.t. $\mathrm{Sj}$, several candidates [i.e., pink circles in (b)] can be found, which are also located at the crowns of MFG, PrG, and PoG, respectively. The same coordinates of these three candidate points are also shown on the image of $\mathrm{Si}$, with dashed pink circles in (a) and(c), respectively. Because each of these three candidates has the similar attribute vector to the voxel $\mathrm{xi}$, a deterministic correspondence detection strategy would lead to mismatch (e.g., xi will be matched to the closest candidate at the crown of PoG). In contrast, soft assignment can help voxel $x i$ find the true correspondence with the guidance of the mean location of all candidates in $\mathrm{Sj}$, as designated by the red arrow. After several iterations, voxel xi will move to gi(xi) [such as a pink dashed circle in (c)]. At that time, one-to-one correspondence is deterministic to achieve the good registration result. [Color figure can be viewed in the online issue, which is available at wileyonlinelibrary.com.] 
Step 1: Determine the spatial correspondence

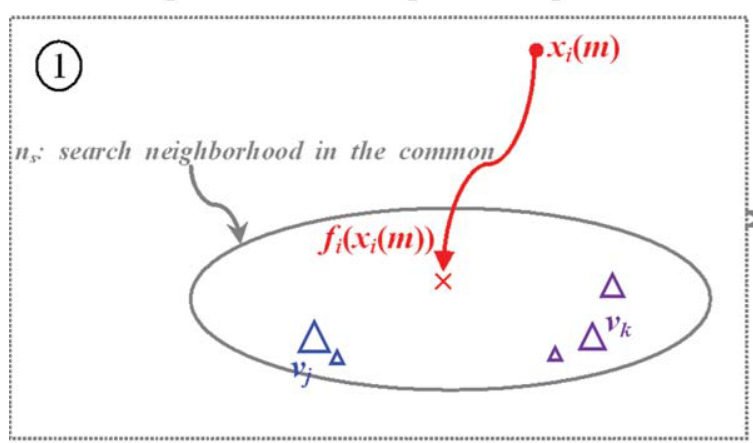

$\times x_{i}(m)$ in the common space

$\Delta$ The candidate correspondence of $x_{i}(m)$ in each subject subject

$\Delta$ The mean correspondence of $x_{i}(m)$ in each subject

(;) Estimated correspondence of $x_{i}(m)$ w.r.t. all other subjects

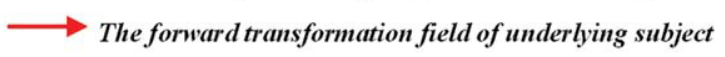

$4---$ The backward transformation field of underlying subject
Step 2: Determine the contribution of each subject

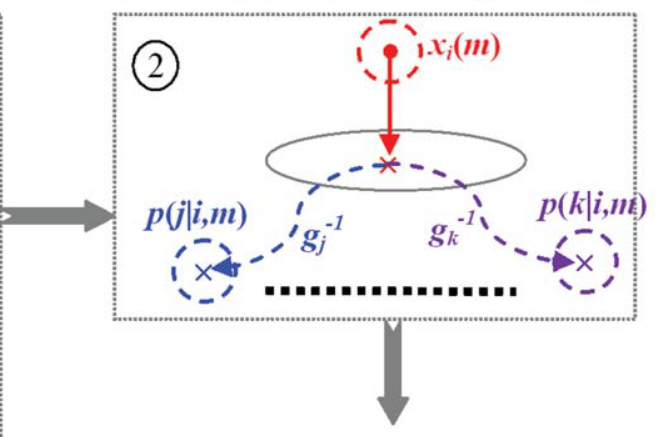

Step 3: Determine the final correspondence w.r.t. all subjects

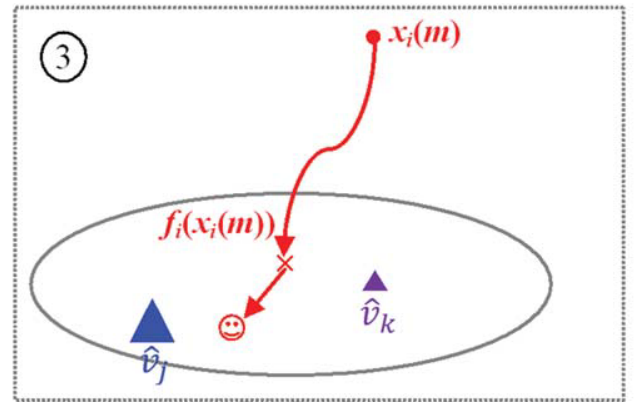

Figure 4.

Illustration of the correspondence detection process in our groupwise registration framework. The different sizes of triangles denote weights in calculating the mean correspondence. See detailed description in the end of "Solution to the Energy Function" Section. [Color figure can be viewed in the online issue, which is available at wileyonlinelibrary.com.]

be implausible for an exact one-to-one correspondence strategy to work in this situation. For example, according to the principle of the iterated closest point (ICP) algorithm [Besl and McKay, 1992], the closest candidate from PoG would be regarded as the correspondence to the voxel $x_{i}$, and then the PrG in $S_{i}$ would be incorrectly warped toward the PoG in $S_{j}$. Fortunately, this problem can be avoided by allowing fuzzy correspondence detection in the beginning of registration and, gradually, progressing to the deterministic correspondence detection in the end of registration. Therefore, all possible candidates will be considered, although their contributions are dynamically adjusted during the registration. Initially, even the faraway voxels will possibly act as candidate correspondences (e.g., the farthest candidate from MFG in $S_{j}$ ). The soft assignment can thus warp voxel $x_{i}$ following the weighted mean direction (designated by the red arrow in Fig. 3a), which is conservative but robust. It is worth noting that the weight for each candidate is adaptively determined according to both the anatomical similarity and the geometric distance to the underlying voxel (see Fig. 4 for demonstration). In this way, after several iterations of registration, the ambiguities in correspondence detection are greatly reduced for most of anatomical structures. Figure $3 \mathrm{c}$ shows the tentative warping result of $S_{i}$, where voxel $x_{i}$ is warped to $g_{i}\left(x_{i}\right)$ (a dashed red circle in (c)) and the MFG, PrG, and PoG are almost matched with the corresponding anatomical structures in $S_{j}$, although more local refinements are required. (Note that the registration is performed in the $3 \mathrm{D}$ image, whereas only cross-sectional views are provided in Fig. 3; therefore, the structures shown in Fig. 3a,c are not necessarily identical to each other.) After initial registration, the voxel at the crown of PrG gyrus in $S_{j}$ is now the nearest one to voxel $x_{i}$ in $S_{i}$ and thus even a deterministic correspondence detection strategy becomes effective by considering only the nearby voxel with the lowest regionwise difference.

\section{Fuzzy Control of Soft Assignment}

According to the demonstration in Figure 3, soft correspondence is especially important in the beginning of registration because it can help avoid the over-dependence on one specific correspondence detected and hence reduces the risk of misguidance. Therefore, for a given driving voxel $x_{i}(m)$ under consideration, each voxel in the search neighborhood $n_{s}$ will be examined. We use the conditional probability $p(v \mid j, i, m)$ to indicate the likelihood of possible correspondence candidate $v \in \Omega_{c}$ for the driving voxel $x_{i}(m)$ when considering only with subject $S_{j}$. The probability value $p(v \mid j, i, m)$ depends on the matching 
criteria [Eq. (9) in next subsection] of updating $f\left(x_{i}(m)\right)$ to $v$. Thus, even the voxels with relatively large discrepancies will still have the chance to become candidate correspondences, thus alleviating the ambiguities in correspondence detection. However, to increase the registration accuracy and specificity, it is necessary to determine one-to-one correspondence in the final stages of registration (after all subjects are mostly aligned). The dynamic control on the soft assignment (from fuzzy to deterministic) is then formulated by requiring the entropy of $p(v \mid j, i, m)$ to decrease gradually with the progress of registration. Denoting $P_{s}=$ $\left\{p(v \mid j, i, m) \mid \in n_{s}, j=1, \ldots, N, j \neq i\right\}$ as the collection of $p(v \mid j, i, m)$, the fuzziness on spatial assignments is:

$F_{s}\left(P_{s}\right)=\sum_{i=1}^{N} \sum_{j=1, j \neq i}^{N}\left\{\sum_{m=1}^{M_{i}} \sum_{v \in n_{s}\left(f_{i}\left(x_{i}(m)\right)\right)}-p(v \mid j, i, m) \cdot \log (p(v \mid j, i, m))\right\}$.

As a groupwise registration method, the correspondences of one subject are required to be determined w.r.t. all other subjects in the group. However, the contribution of each subject is different and will dynamically change throughout the registration. In our method, the rough-tofine strategy used in detecting spatial correspondence is also used when selecting subjects for correspondence detection. Similarly, we use the conditional probability $p(j \mid i, m)$ to describe the likelihood of subject $S_{j}$ being a candidate for correspondence detection of $x_{i}(m)$, according to the similarity of local anatomies (see the dashed circles in Fig. 4) in $S_{i}$ and $S_{j}$. It is worth noting that the role of subject assignment is very important because it makes the registration robust to possible outliers in the population of subjects [Fletcher et al., 2009]. As we will explain in Eq. (12) later, in the beginning of registration, every subject contributes equally in determining the correspondence for $x_{i}(m)$ to capture the global characteristics within the population. As the registration progresses, all subjects are progressed to the common space. At that time, the subjects, which are very different on local anatomies, should influence less or even be excluded in the correspondence detection. Denoting $P_{p}=\{p(j \mid i, m) \mid j=1, \ldots, N, j \neq i\}$ as the collection of $p(j \mid i, m)$, the fuzziness on subject assignment can be defined as:

$$
F_{P}\left(P_{P}\right)=\sum_{i=1}^{N} \sum_{m=1}^{M_{i}}\left\{\sum_{j=1, j \neq i}^{N}-p(j \mid i, m) \cdot \log (p(j \mid i, m))\right\}
$$

The solutions for $p(v \mid j, i, m)$ and $p(j \mid i, m)$ will be provide below.

\section{Improved Energy Function in SP,}

To integrate the above-described strategies, we refine the energy function $E_{1}(F)$ in three ways. First, we consider the problem of correspondence detection in the domain of common space $\Omega_{c}$, because it can simplify the procedure of feature matching in groupwise scenario by always taking $\Omega_{c}$ as the reference. Second, when refining the correspondence on the driving voxel $x_{i}(m)$, we will visit each voxel $v$ in the neighborhood $n_{s} \subset \Omega_{c}$, where the center of $n_{s}$ is designated by $f_{i}\left(x_{i}(m)\right)$ and the resulting update is $\Delta f_{i}\left(x_{i}\right)=v-f_{i}\left(x_{i}\right)$. The criteria for evaluating the qualified candidates $v$ are similar with that in the brace of Eq. (3): (1) the candidate location $v$ in $n_{s}$ should have low regionwise difference $D_{i \rightarrow j}\left(\vec{a}_{i}\left(x_{i}\right), \Delta f_{i}\left(x_{i}\right)\right)$ [Eq. (6)] w.r.t. Subject $S_{j}$; (2) the geometric distance between previously estimated $f_{i}\left(x_{i}(m)\right)$ and the candidate location $v$, that is, $\left\|\Delta f_{i}\left(x_{i}(m)\right)\right\|^{2}$, should be as close as possible, according to the ICP principle [Besl and McKay, 1992]. Based on these criteria, the risk of updating the correspondence from the previously estimated $f_{i}\left(x_{i}(m)\right)$ to the new location $v$ w.r.t. the particular subject $S_{j}$ can be formulated as:

$$
\xi_{i \rightarrow j}\left(\Delta f_{i}\left(x_{i}\right)\right)=D_{i \rightarrow j}\left(\vec{a}_{i}\left(x_{i}\right), \Delta f_{i}\left(x_{i}(m)\right)\right)+\left\|\Delta f_{i}\left(x_{i}(m)\right)\right\|^{2} .
$$

Third, we also adopt the soft assignment strategy to our groupwise registration algorithm (due to its importance in robust correspondence), in a similar way as we have done for brain MR image registration in [Shen, 2009] and DTI image registration in [Yap et al., 2009]. Deterministic oneto-one correspondence strategy will only select the candidate $v$ with the minimal $\xi_{i \rightarrow j}\left(\Delta f_{i}\left(x_{i}\right)\right)$, which might misguide the registration in the beginning as demonstrated above. On the other hand, soft assignment is able to alleviate this obstacle by considering all possible candidates and then weighting each candidate with its conditional probabilities $p(v \mid j, i, m)$ in the spatial domain and $p(j \mid i, m)$ in the population domain.

By integrating these three strategies into the correspondence detection, we can formulate the energy function in $S P_{1}$ as:

$$
\begin{array}{r}
E_{1}\left(\hat{F}, P_{s}, P_{P}\right) \\
=\left\{\sum_{i=1}^{N}\left[\sum_{m=1}^{M_{i}} \sum_{v \in n_{s}\left(f_{i}\left(x_{i}(m)\right)\right)} p(v \mid j, i, m) \cdot p(j \mid i, m) \zeta_{i \rightarrow j}\left(\Delta f_{i}\left(x_{i}\right)\right)\right]\right. \\
\left.-r_{1} \cdot F_{s}\left(P_{s}\right)-r_{2} \cdot F_{P}\left(P_{P}\right)\right\}
\end{array}
$$

where $r_{1}$ and $r_{2}$ are two positive scalars that control the fuzziness of spatial assignment and subject assignment, respectively. We allow multiple correspondences for each driving voxel $x_{i}(m)$ by assigning a probability $p(v \mid j, i, m)$ to measure the spatial distance and $p(j \mid i, m)$ to describe the local affinity with other subjects in population. To dynamically control the fuzziness of correspondences, we require the distribution of $p(v \mid j, i, m)$ and $p(j \mid i, m)$ to change from uniform to spiky, which is achieved by minimizing the entropy terms $F_{s}\left(P_{s}\right)$ in Eq. (7) and $F_{P}\left(P_{P}\right)$ in Eq. (8), respectively. We will detail the solution to Eq. (10) next. 


\section{Solution to the Energy Function}

To optimize the energy function in Eq. (10), we iteratively use the local greedy search strategy to update the correspondence $f_{i}\left(x_{i}(m)\right)$ on each driving voxel $x_{i}(m)$ in two steps. In the first step, we calculate the probability of $p(v \mid j, i, m)$ and $p(j \mid i, m)$ by letting $\partial E_{1} / \partial p(v \mid j, i, m)=0$ and $\partial E_{1} / \partial p(j \mid i, m)=0$. Then the new correspondence estimation $f_{i}\left(x_{i}(m)\right)$ can be obtained with the guidance of $p(v \mid j, i, m)$ and $p(j \mid i, m)$, which indicates the likelihood of each candidate location $v$ w.r.t. subject $S_{j}$ and the contribution of $S_{j}$ in the feature matching.

The explicit solutions to $P_{S}$ and $P_{P}$ can be obtained by letting $\partial E_{1} / \partial p(v \mid j, i, m)=0$ and $\partial E_{1} / \partial p(j \mid i, m)=0$, as shown in Eqs. (11) and (12) below:

$$
p(v \mid j, i, m)=c_{1} \cdot e^{-\frac{\zeta_{i \rightarrow j}\left(\Delta f_{i}\left(x_{i}(m)\right)\right)}{r_{1}}}, \quad p(v \mid j, i, m) \leftarrow \frac{p(v \mid j, i, m)}{\sum_{v} p(v \mid j, i, m)},
$$

$$
\begin{aligned}
& p(j \mid i, m)
\end{aligned}
$$

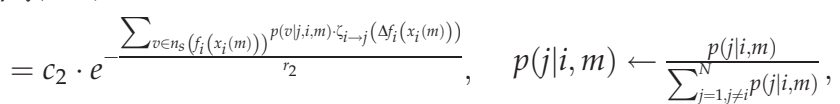

where $c_{1}$ and $c_{2}$ denote constants. The normalization is necessary to satisfy that $0 \leq p(v \mid j, i, m) \leq 1$ and $0 \leq$ $p(j \mid i, m) \leq 1$.

The degree of soft assignment is controlled by the parameter $r_{1}$ and $r_{2}$, which act as the role of temperature in the simulated annealing scenario. Notice that $r_{1}$ penalizes the large risk $\left(\xi_{i} \rightarrow{ }_{j}\left(\Delta f_{i}\left(x_{i}\right)\right)\right)$ of feature matching for one candidate location $v$, and $r_{2}$ penalizes the overall discrepancy $\left(\sum_{v \in n_{s}(f i(x i(m)))} p(v \mid j, i, m) \cdot \xi_{i \rightarrow j}\left(\Delta f_{i}\left(x_{i}\right)\right)\right)$ within $n_{s}$, both in the exponential way. Therefore, when $r_{1}$ and $r_{2}$ have high values, Eqs. (11) and (12) will enforce a more uniform distribution (on both $p(v \mid j, i, m)$ and $p(j \mid i, m)$ ) and, hence, encourage correspondence detection to look for multiple correspondences in multiple subjects, thus making the algorithm more robust to ambiguity. When $r_{1}$ and $r_{2}$ have low values, a more constricted impulse-like distribution will be enforced, thus yielding a stricter oneto-one correspondence within a few most similar subjects. In our algorithm, $r_{1}$ and $r_{2}$ are progressively decreased, as described in [Chui and Rangarajan, 2003]. It is worth noting that the influences of $r_{1}$ and $r_{2}$ are slightly different in that the spatial assignment is required to turn to the deterministic assignment in the end of registration, whereas the subject assignment is much less restricted, that is, all the subjects will be taken into consideration although some of them might be less considered in the end.

Recalling that $\Delta f_{i}\left(x_{i}\right)=v-f_{i}\left(x_{i}\right)$ and removing unrelated variables with $f_{i}\left(x_{i}(m)\right)$ in Eq. (10), the new correspondence $\hat{f}_{i}\left(x_{i}(m)\right)$ on each driving voxel $x_{i}(m)$ can be computed by minimizing $E_{1}$ w.r.t. $f_{i}\left(x_{i}(m)\right)$ :

$$
\begin{aligned}
\hat{f}_{i}\left(x_{i}(m)\right)=\hat{v}=\sum_{j=1, j \neq i}^{N} p(j \mid i, m) \cdot \hat{v}_{j} \\
=\sum_{j=1, j \neq i}^{N} p(j \mid i, m) \cdot\left\{\sum_{v \in n_{s}\left(f_{i}\left(x_{i}(m)\right)\right)} p(v \mid j, i, m) \cdot v\right\},
\end{aligned}
$$

where $\hat{v}_{j}$ denotes the spatial mean location of correspondence w.r.t. $S_{j}$, and $\hat{v}$ is the destination of $x_{i}(m)$ in the common space after evaluating all the mean locations $\hat{v}_{j}$ in all subjects $S_{j}$ (see Fig. 4). It is worth noting that the multiplication of $p(v \mid j, i, m)$ and $p(j \mid i, m)$ produces the conditional probability $p(v, j \mid i, m)$, which can be used as a guidance in our registration algorithm to determine the destination of $x_{i}(m)$ in the common space.

Figure 4 demonstrates the procedure of refining the transformation on $x_{i}(m)$ in subject $S_{i}$ w.r.t. the rest subjects in the group. The refinement is performed in the common space in three steps, as shown in Figure 4. In our groupwise registration method, the update of correspondence of the driving voxel $x_{i}(m)$ (the red ' $\times$ ' in Fig. 4) is required to consider all the other subjects, where we use different colors to denote candidate correspondences in different subjects (e.g., blue and purple in Fig. 4). In the first step, the regionwise difference of each possible candidate $v_{j}$ in the search neighborhood $n_{s}$ will be evaluated w.r.t. a particular subject $S_{j}$, where the calculation of regionwise difference can be referred in Section "Regionwise Feature Matching" and Figure 2. As the result, each candidate $v_{j}$ has been associated with the posteriori probability $p(v \mid j, i, m)$ to indicate the likelihood of true correspondence [by Eq. (11)]. Here, we use the different size of the unfilled triangles to represent the degree of $p(v \mid j, i, m)$. Note that the same procedure will be performed to all the subjects other than $S_{i}$. In the second step, the priori probability $p(j \mid i, m)$ will be determined [see Eq. (12)] by calculating the regionwise different between $x_{i}(m)$ and its correspondences $g_{j}^{-1}\left(f_{i}\left(x_{i}(m)\right)\right)$ in the subject $S_{j}$. In the third step, the mean correspondence location in each subject (e.g., $v_{j}$ and $\hat{v}_{k}$ w.r.t. subject $S_{j}$ and subject $S_{k}$, respectively) will be obtained according to the formulation in the bracket of Eq. (13). Then, based on the priori probability $p(j \mid i, m)$, where the degree of $p(j \mid i, m)$ is indicated by the size of the solid triangles, the new estimated correspondence $\widehat{f}_{i}\left(x_{i}(m)\right)$ will point to the red smile face in Figure 4 , which is the weighted average of all $\hat{v}_{j}$ s.

\section{SUMMARY AND IMPLEMENTATION ISSUES}

\section{Summary of Our Groupwise Registration Algorithm}

Our groupwise registration algorithm is briefly summarized below:

1. Calculate attribute vectors for each subject $S_{i}$;

2. Set $g_{i}(x)=x$, and $g_{i}^{-1}=x, \forall i \in 1, \ldots, N$; 

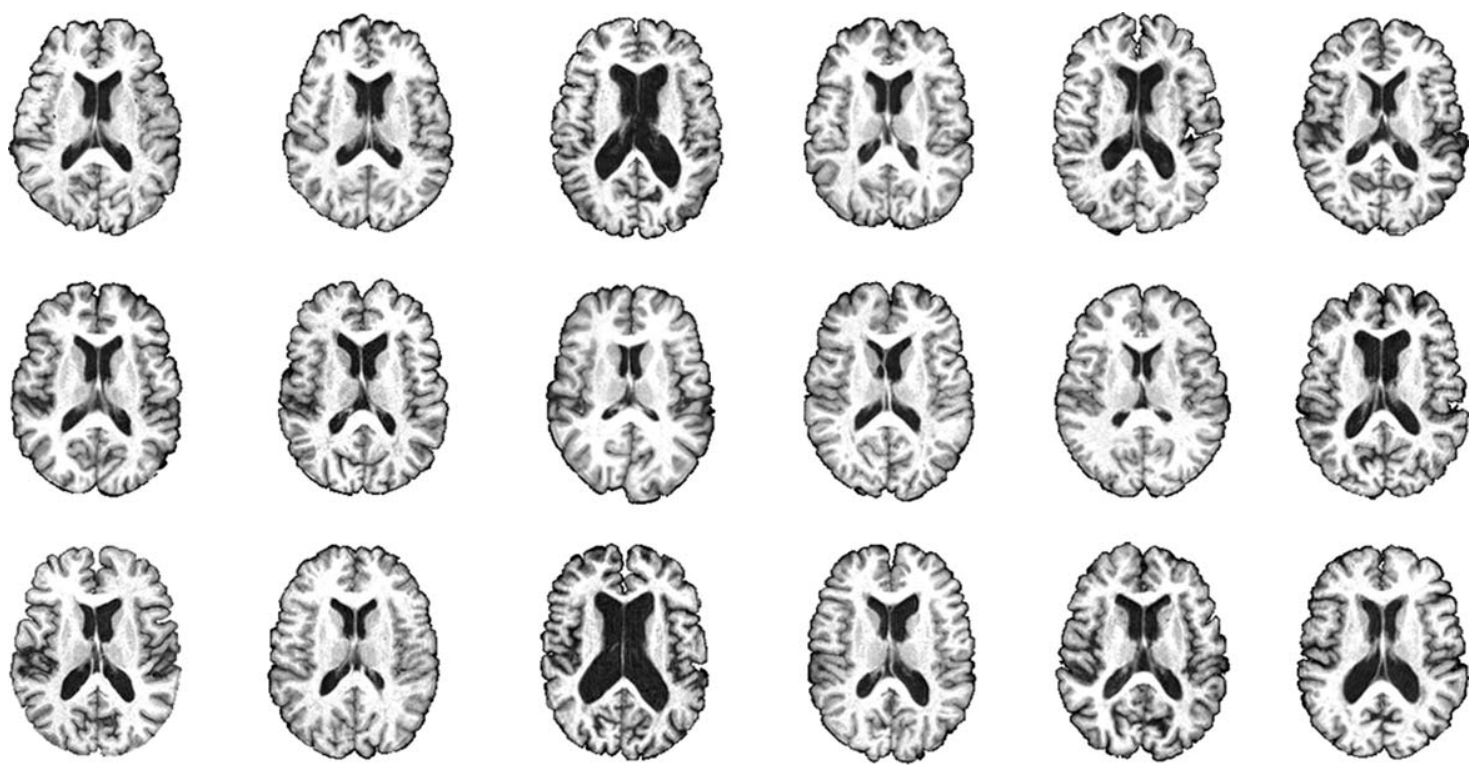

Figure 5.

Eighteen elderly brain images used in evaluation of three registration methods. Each subject will be selected as a template image, one by one, for the pairwise registration algorithm to fairly evaluate its performance.

3. Select a current set of driving voxels $\mathrm{DV}_{i}$ for each subject $S_{i}$ according to a certain criterion [Shen and Davatzikos, 2002];

4. Set $f_{i} \leftarrow g_{i}$ on each subject $S_{i}$;

5. For each subject $S_{i}$, fix $g_{i}^{-1}$ and do the following:

1. Refine the groupwise correspondence for each driving voxel $x_{i}(m)$ using a soft assignment strategy, and then obtain $f_{i}\left(x_{i}(m)\right)$ ("Regionwise Feature Matching, Soft Correspondence Assignment, and Fuzzy Control of Soft Assignment" Sections);

2. Use the current estimated correspondence field $\widehat{f}_{i}$ on the driving voxels to interpolate the dense transformation field $\widehat{g}_{i}$ by TPS [Bookstein, 1989a].

3. Normalize the estimated transformations using $\hat{g}_{i}(x) \leftarrow\left[\hat{g}_{i}(x)-\sum_{i=1}^{N} \hat{g}_{i}(x) / N\right]$, to satisfy the global drift constraint in Eq. (4);

6. Reverse the dense transformation field $g_{i}$ and obtain $g_{i}^{-1}$ using a technique developed in Section II.C of [Christensen and Johnson, 2001];

7. If not converged (e.g., not reaching the number of predetermined iterations or the energy function is changed less than a predefined threshold), relax the criterion on selecting the driving voxels and go to the step 3).

\section{Implementation Issues}

We introduce a multiresolution strategy to implement our proposed groupwise registration method for fast and robust registration. Specifically, in each resolution, the size of the search neighborhood $n_{s}$ decreases gradually with the progress of registration (e.g., the radius of $n_{s}$ is decreased from 12 to 1 in the fine resolution), for achieving more specific detection of correspondences. Moreover, in the initial state of registration, only a small set of voxels ( $2-3 \%$ of the whole image voxels) with distinctive features, such as those located at ventricular boundaries, sulcal roots, and gyral crowns, are selected as driving voxels. After that more and more driving voxels are added to drive the registration and eventually all voxels in the brains join the groupwise registration.

With the increase of the number of driving voxels during the groupwise registration, the TPS-based interpolation becomes burdensome. Accordingly, we turn to use TPS for the small overlapping blocks (with size of $32 \times 32 \times 32$ ) and also down-sample the driving voxels in each of these blocks, that is, with less than 500 driving voxels finally selected as control points for each block. In this way, we can efficiently interpolate the dense deformation in the whole image domain by the TPS.

\section{EXPERIMENTS}

In our experiments, we have extensively evaluated the performance of our groupwise registration method in atlas construction, ROI labeling, and brain atrophy detection. For comparison, we use a congealing groupwise registration method implemented by Balci [Balci et al., 2007], with open sources available (http://www.insight-journal.org/ browse/publication/173). To demonstrate the advantage 


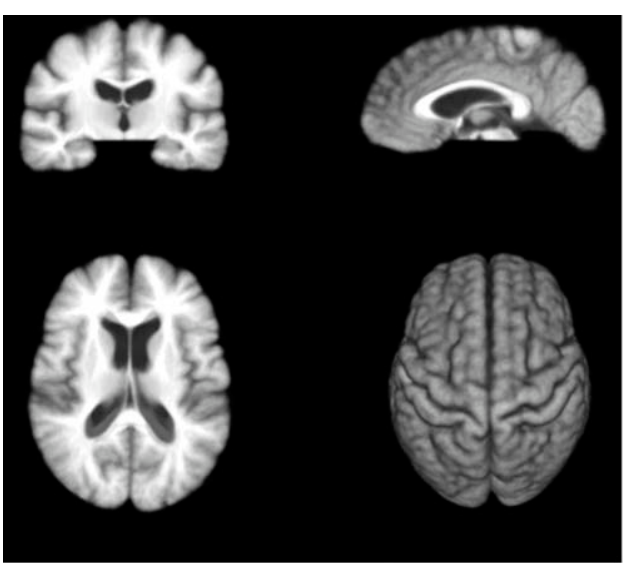

(a) The group mean image by congealing method

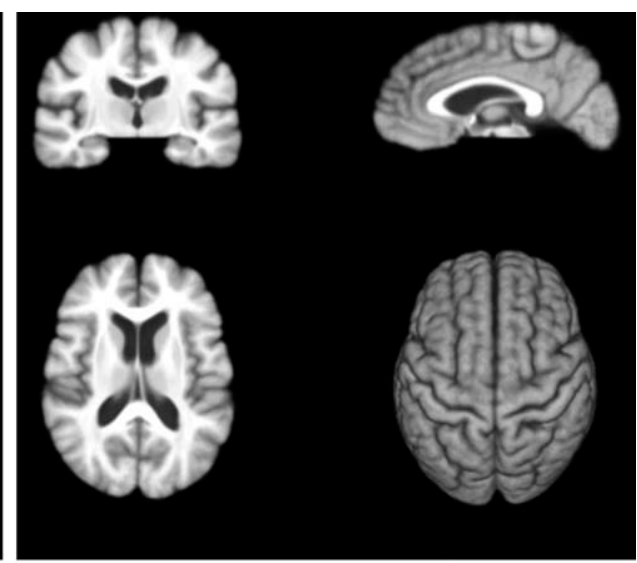

(b) The group mean image by our method

Figure 6.

The groupwise registration results by the congealing method and our method. It can be observed that our group mean image is sharper than that by the congealing method, indicating a more accurate and consistent registration by our method.

of groupwise registration compared with pairwise registration, the registration results by a pairwise registration method, such as HAMMER [Shen and Davatzikos, 2002], are also provided. For real brain images, we first evaluate the group mean image and the overlap ratios on WM, GM, and ventricle (VN) for 18 elderly subjects. Then, we compare the overlap ratios of aligned ROIs using NIREP dataset (http://www.nirep.org/) and LPBA40 dataset (http://www.loni.ucla.edu/Atlases/LPBA40), which have 32 and 54 manually delineated labels for each brain, respectively. We also compare the performance of three registration methods in helping detect the group difference between 12 pairs of normal controls and simulated atrophic subjects.

The overlap ratio of ROIs is an important criterion to demonstrate the registration performance. Here, we use the Jaccard Coefficient metric [Jaccard, 1912] to measure the alignment of two regions with same label. For the two registered regions $\mathrm{A}$ and $\mathrm{B}$, the Jaccard Coefficient is defined as:

$$
J(A, B)=\frac{|A \cap B|}{|A \cup B|},
$$

where $|$.$| denotes the number of voxels in the underlying$ region. For demonstrating the group overlap of brain images after registration, we specifically vote a reference by assigning each voxel with a tissue label that is the majority of all tissue labels at the same location from all the aligned subjects. Then the Jaccard Coefficient between each of the registered label images and the voted reference will be calculated. It is worth noting that this is a very strict definition for measurement of ROI overlap, but it emphasizes the importance of groupwise registration in measuring the group performance. In the following experi- ments, we use the average score of Jaccard Coefficients as the overlap ratio for each tissue label.

\section{Experiment on I 8 Elderly Brains}

Figure 5 shows a group of 18 elderly brain images. Each image has the size of $256 \times 256 \times 124$ and the resolution of $0.9375 \times 0.9375 \times 1.5 \mathrm{~mm}^{3}$. It can be observed that the anatomical structures vary largely across different subjects, especially for the ventricle and the cortex.

The group mean images produced by the congealing method, and our groupwise registration method are both displayed in Figure 6. Through visual inspection, our group mean image is sharper and gains better contrast (especially around ventricles) than that by the congealing method, indicating higher performance of our registration method.

The overlap ratios on WM, GM, and VN, as well as the overall overlap ratio on whole brain, by our method and the congealing method are displayed in Table I. It can be observed that our method achieves better results than the congealing method, that is, our method achieves overall overlap ratio of $72.05 \%$, whereas the congealing method achieves $59.43 \%$. By checking the overlap ratio of each individual tissue type, our results also outperform the congealing method in all brain tissues. To further quantitatively evaluate the registration consistency, we calculate the entropy of tissue probability on each voxel across 18 aligned subjects. The average entropy value is 0.105 by our method, much lower than 0.138 by the congealing method. Note that the stack entropy on each voxel is explicitly minimized in the congealing algorithm. Even in this case, our method performs better, although we did not explicitly minimize it in our algorithm. On the other 
- Feature-Based Groupwise Registration •

TABLE I. Overall overlap ratios of WM, GM, and VN by pairwise HAMMER algorithm, congealing method, and our groupwise registration method

\begin{tabular}{lcccc}
\hline & WM & GM & VN & Overall \\
\hline Pairwise HAMMER & $64.05 \%( \pm 4.13 \%)$ & $57.11 \%( \pm 2.56 \%)$ & $75.46 \%( \pm 4.08 \%)$ & $65.26 \%( \pm 3.52 \%)$ \\
Congealing Method & $59.68 \%$ & $51.09 \%$ & $70.61 \%$ & $59.43 \%$ \\
Our Method & $75.81 \%$ & $63.61 \%$ & $81.16 \%$ & $72.05 \%$ \\
\hline
\end{tabular}

hand, it is interesting to compare the performance between groupwise and pairwise registrations of these 18 brain images. In HAMMER-based pairwise registration, each subject is selected as the template, one by one, to align with all other 17 remaining subjects. The average overlap ratios produced, as well as the standard deviations, are shown in the first row of Table I, which verifies again the power of our groupwise registration in consistently registering the population images.

\section{Experiment on NIREP Data}

In this experiment, we align 16 brain subjects in NIREP dataset [Christensen et al., 2006] by both congealing and

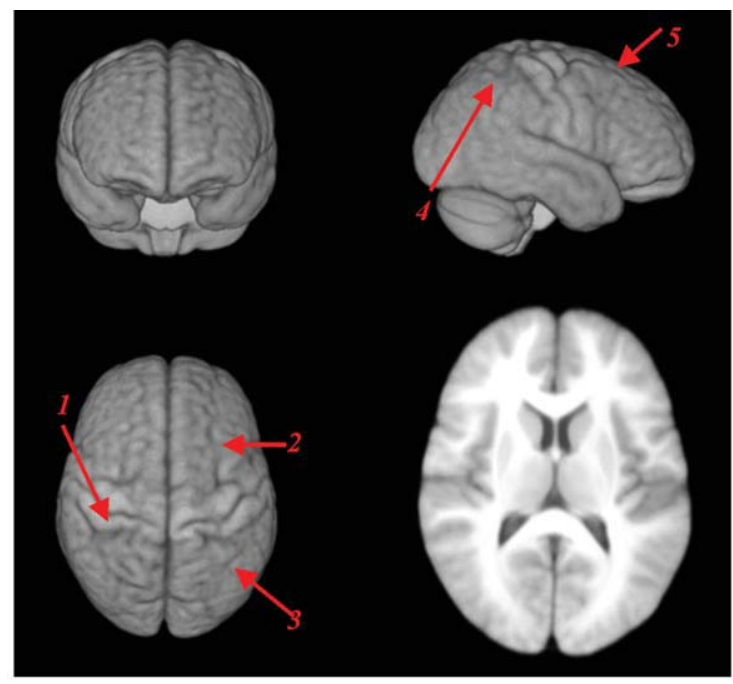

(a) The group mean image by congealing method

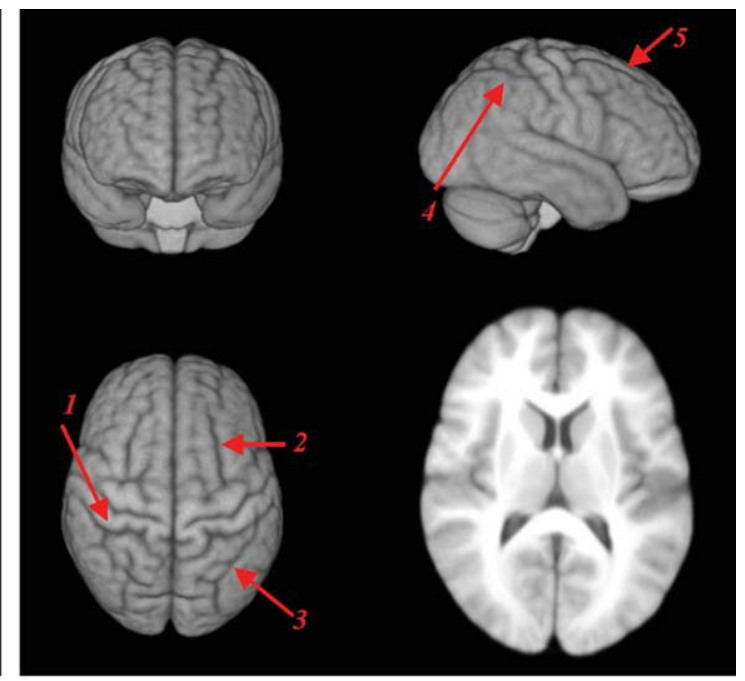

(b) The group mean image by our method
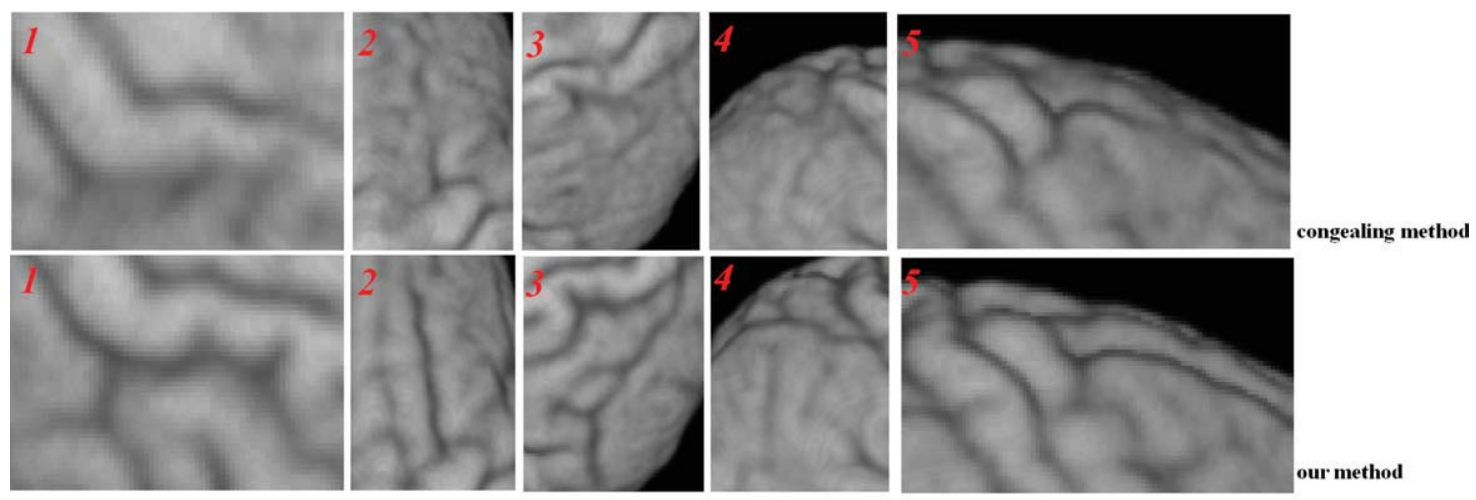

congealing method

(c) The zoom-in view of group mean images

Figure 7.

The group mean image of 16 NIREP brain images. The group mean images by the congealing method and our method are displayed in the left and right panels, respectively. Through visual inspection, our group mean image is sharper than that produced by the congealing method, especially in the cortex as indicated by arrows (with the zoomed views shown in the bottom). [Color figure can be viewed in the online issue, which is available at wileyonlinelibrary.com.] 


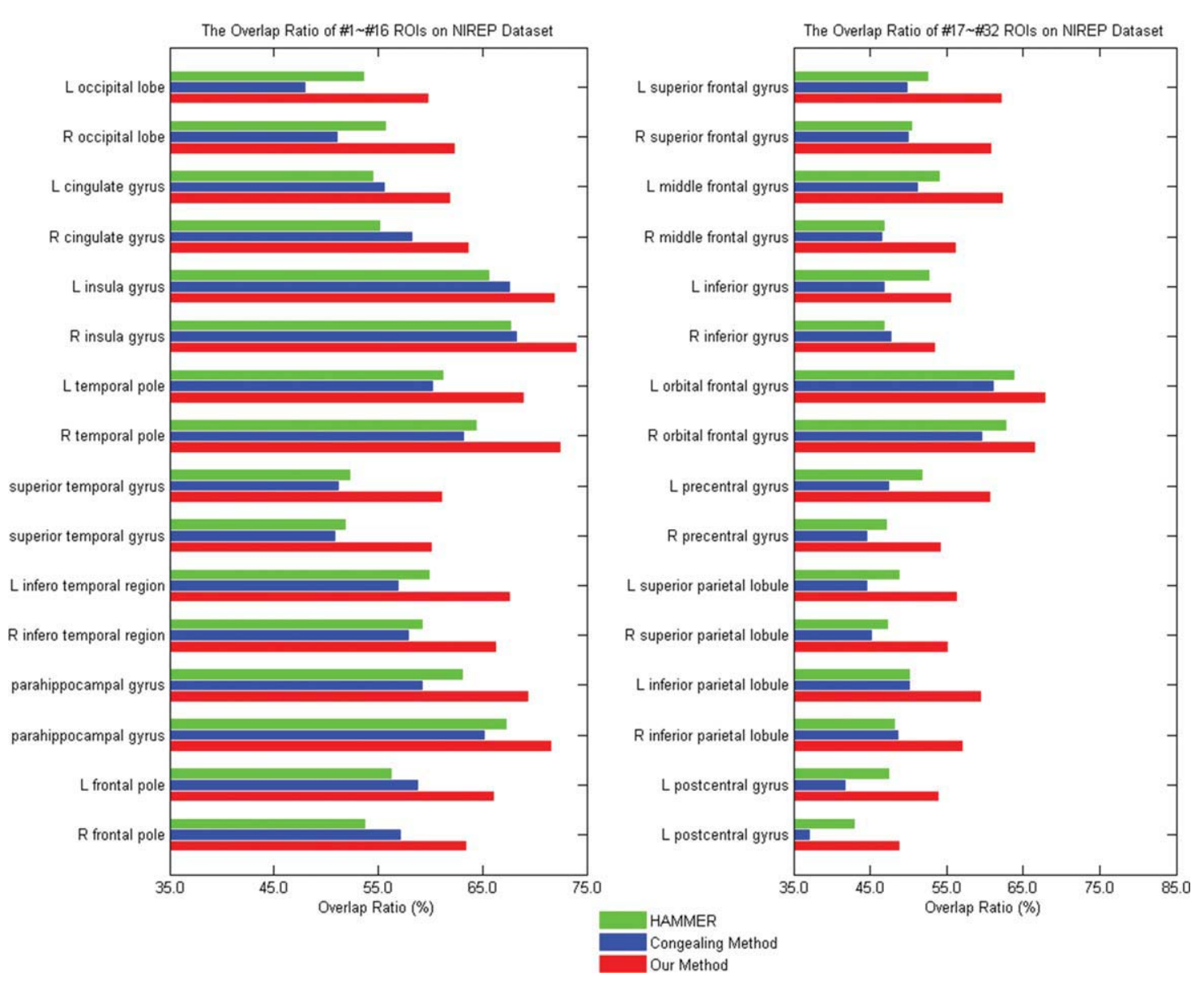

Figure 8.

The overlap ratios of 32 manually delineated labels in 16 NIREP brain images. The overlap ratios by HAMMER, congealing method, and our groupwise registration method are shown in green, blue, and red, respectively. It can be observed that our method achieves the best alignment result in most ROI regions. The overall overlap ratio is $56.58 \%$ by HAMMER, $52.07 \%$ by the congealing method, and $61.52 \%$ by our method. [Color figure can be viewed in the online issue, which is available at wileyonlinelibrary.com.]

our groupwise registration methods, respectively. The NIREP dataset consists of 16 3D MR images of eight normal male and eight normal female adults, each with 32 manually delineated GM ROIs. All 16 MR images have been aligned according to the anterior and posterior commissures (AC and PC). The image size is $256 \times 300 \times 256$, and the voxel dimension is $0.7 \times 0.70 .7 \mathrm{~mm}^{3}$. The group mean images produced by the congealing method and our registration method are displayed in the left and right panel of Figure 7, respectively. It can be observed that our group mean image is not only sharper than that by the congealing method but also gains much more anatomical details in the mean image, especially in the cortex as indicated by arrows in Figure 7. To have a better comparison, we zoom up the five regions (as indicated by the red arrows in Fig. 7a) and show them in Figure 7c.
To show the advantage of groupwise registration over pairwise registration, we also use HAMMER to align 16 subjects by selecting one subject as a template. Figure 8 displays the overlap ratio on each ROI by pairwise HAMMER, congealing method, and our groupwise registration method. The overall overlap ratio is $56.58 \%$ by HAMMER, $52.07 \%$ by congealing method, and $61.52 \%$ by our groupwise method. For the two groupwise registration methods, the results by our method are much better than that by the congealing method, in terms of the overall overlap ratio, as well as the individual overlap ratio in each ROI except the right cingulated gyrus and right inferior gyrus. After checking the warping result at those two gyri, we found that their volume sizes are much smaller compared with their nearby ROIs. Thus, our registration method may underestimate the registration at those two regions 


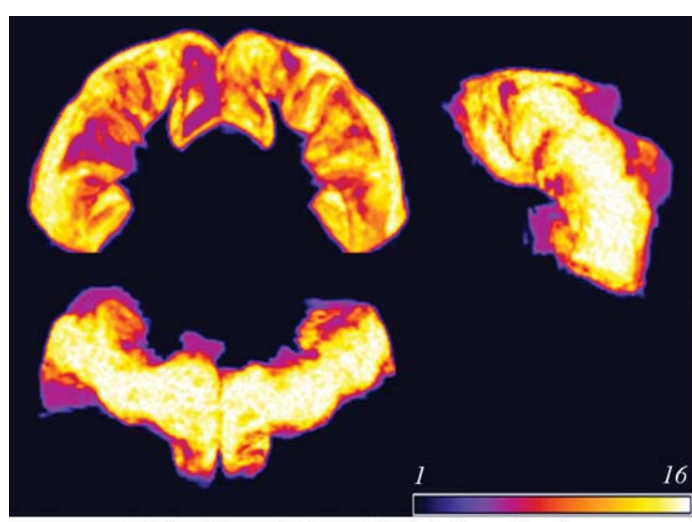

(a) The 3D rendering of the label overlapping

results by the congealing method

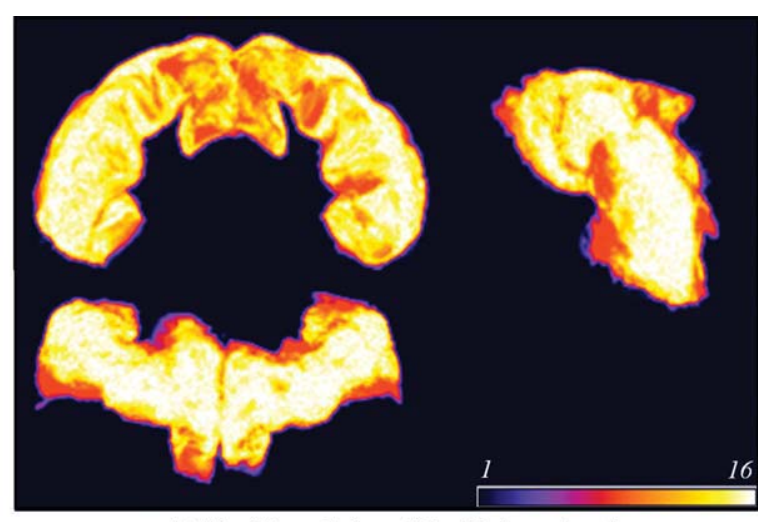

(b) The 3D rendering of the label overlapping

Figure 9.

The 3D renderings of the label overlapping results on the left and right precentral gyri. After groupwise registration by the congealing method or by our method, we can overlap the labels of 16 registered NIREP brain images at the left and right precentral gyri and show their 3D renderings in the left and right panels of this figure, respectively. Bright color indicates better overlap, whereas dark color means poor overlap after registration. [Color figure can be viewed in the online issue, which is available at wileyonlinelibrary.com.]

due to the lack of enough number of driving voxels to steer the registration. On the other hand, our previous attribute-vector guided groupwise registration method [Wang et al., 2010] can produce the overall overlap ratio of $58.86 \%$, indicating again the better performance of our current proposed method.

Figure 9 shows the performance of registration consistency at the left and right precentral gyri by the congealing method and our method. The average overlap ratio is $45.95 \%$ by the congealing method and $57.34 \%$ by our method. The brighter color indicates the higher consistency of registration, whereas the darker color means the poorer overlap ratio, with a color bar provided in the bottom of each panel. It is obvious that our method (as shown in the right panel of Fig. 9) achieves much better alignment for the 16 subjects than the congealing method (as shown in the left panel of Fig. 9).

\section{Experiment on LONI LPBA40 Data}

In this experiment, we use the LONI LPBA40 dataset [Shattuck et al., 2008] with 40 brain images and 54 manually labeled ROIs in each brain image. The image size is $181 \times 217-181$, and the voxel size is $1 \times 1 \times 1 \mathrm{~mm}^{3}$. Similarly, we use pairwise HAMMER, congealing method, and our groupwise registration method to align these 40 brain images. The overall overlap ratio is $54.12 \%$ by HAMMER, $60.60 \%$ by the congealing method, and $67.02 \%$ by our method. This indicates $6.42 \%$ increase by our method, compared with the congealing method. The detailed overlap ratios in each ROI by these three algorithms are provided in Figure 10. Obviously, our groupwise registration method achieves the most accurate and consistent registration results among all three registration methods. On the other hand, our previous attribute-vector guided groupwise registration method [Wang et al., 2010] can produce the overall overlap ratio of $63.26 \%$, which is lower than our current proposed method.

\section{Experiment on Simulated Atrophy Brain Images}

In this experiment, a group of 12 subjects (image size is $256 \times 256 \times 124$ and voxel size is $0.9375 \times 0.9375 \times 1.5$ $\mathrm{mm}^{3}$ ) aged over 65 were selected and simulated atrophy was introduced in both PCG and superior temporal gyrus (STG) of each of these 12 subjects, thus generating another group of 12 atrophic subjects [Davatzikos et al., 2001; Xue et al., 2006] as shown in Figure 11. In principle, the shape and local volumetric difference between individuals or groups can be estimated by the statistical comparisons of shape transformations that map the individual anatomies to the template or common space [Davatzikos et al., 2001]. Two groupwise registration methods, that is, the congealing method and our method, are applied, respectively, to these 24 brain images for simultaneously aligning them onto a common space. Besides, we also use the pairwise HAMMER registration algorithm to align these 24 images onto a selected template.

After aligning these 24 images by each of three registration methods, we can test the capability of each method in detecting atrophy simulated in the precentral and superior temporal gyri. The difference between the normal control group and the simulated atrophy group can be measured by performing a paired $t$-test on the Jacobian determinant 
- Wu et al.
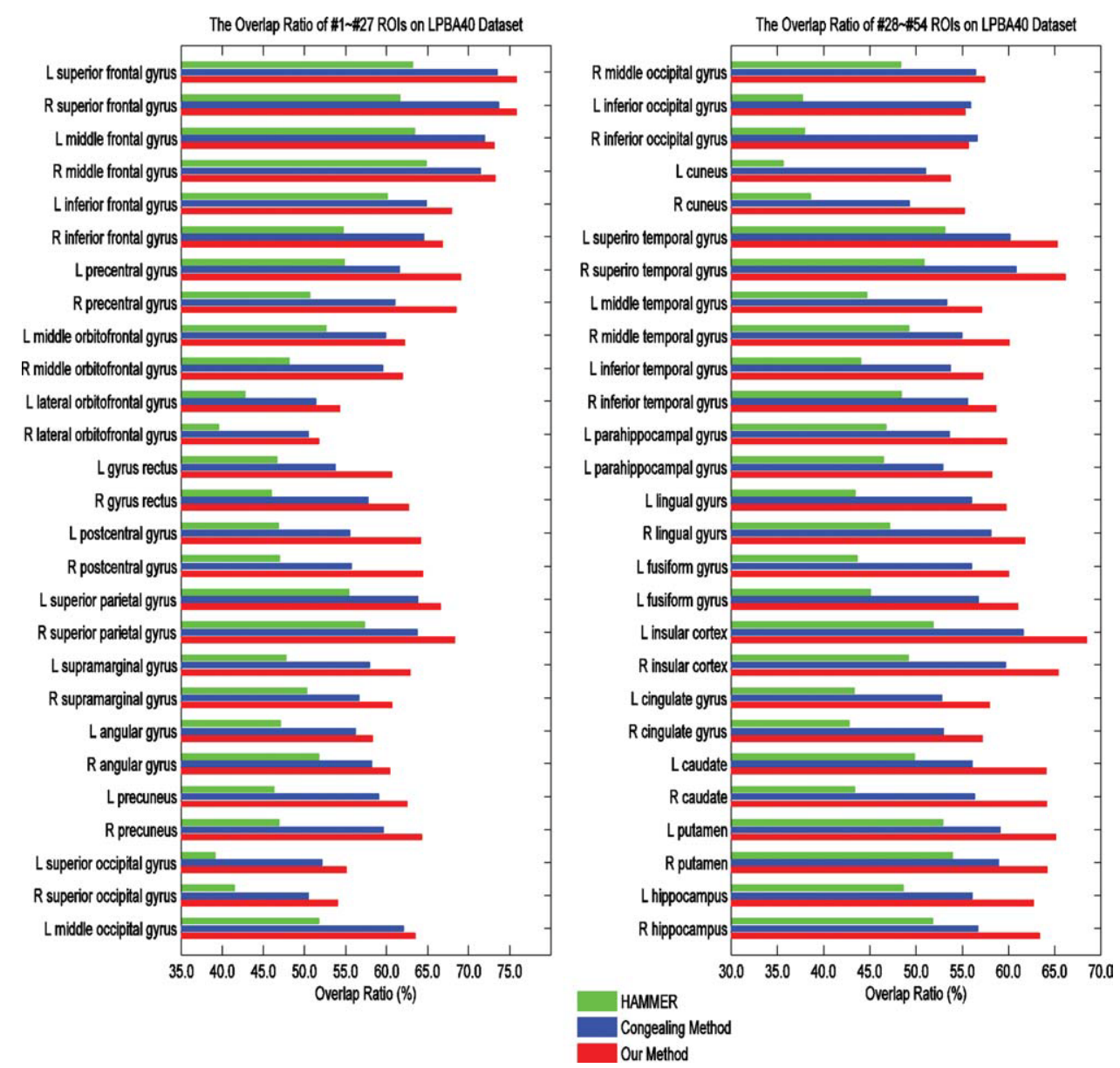

Figure 10.

The overlap ratios of 54 manually delineated ROls in 40 registered LONI LPBA40 brain images. The overlap ratios in each ROI by HAMMER, congealing method, and our groupwise registration method are displayed in green, blue, and red, respectively. As indicated in the figure, our method achieves the best registration results in most ROls. Specifically, the overall overlap

ratio is $54.12 \%$ by HAMMER, $60.60 \%$ by the congealing method, and $67.02 \%$ by our method. This indicates $6.42 \%$ increase by our method, compared with the congealing method. [Color figure can be viewed in the online issue, which is available at wileyonlinelibrary.com.]

maps of the transformation fields estimated for all 24 brain images. Larger $t$-value suggests the better separation (or large difference) between the two groups. We use $P=$ 0.001 as the confidence interval, and the $t$-values obtained by HAMMER, congealing method, and our groupwise registration method are provided in Table II. It can be observed that our method achieves the largest $t$-value among the three methods.

\section{Computation Time}

All of the above experiments are performed on dell work station (with 8 Xeon CPU @2.66 GHz and 32G DDR memory). To speed up the computation time, we use the

OpenMP (www.openmp.org) to parallel the code. The computation times of congealing method and our groupwise registration method are shown in Table III. Both methods use the multithread technique to gain the speed up and have the comparable computation time. But the registration performance by our method is much better than the congealing method.

\section{CONCLUSIONS}

We have presented a groupwise registration method and demonstrated its applications in atlas construction and population data analysis. To address the importance of anatomical correspondence, we propose to use attribute 


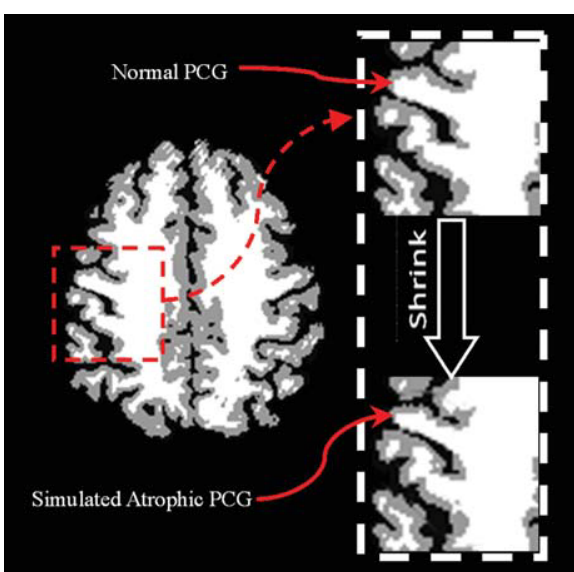

(a) simulated atrophy on the precentral gyrus

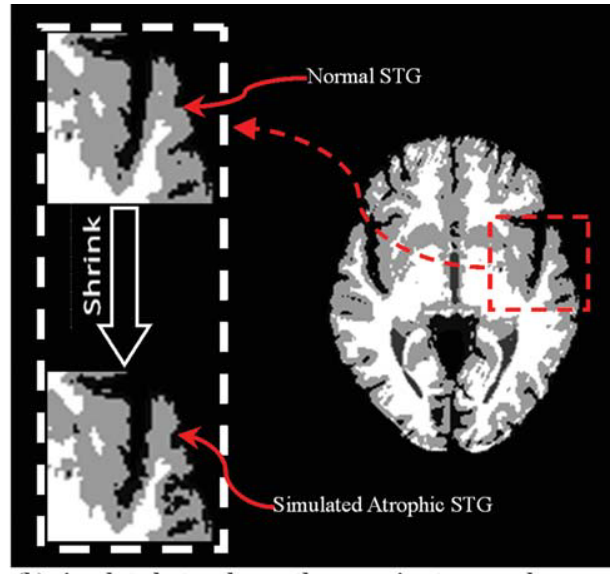

(b) simulated atrophy on the superior temporal gyrus

Figure II.

One example of a pair of normal subject and simulated atrophic subject. Two slices of a normal subject used to simulate atrophy on precentral gyrus (PCG) and superior temporal gyrus (STG) are both displayed in the left and right panels, respectively. To have a better look at the subtle morphological changes (GM shrinkage) around PCG and STG, their zoom-in views are shown in white dashed rectangles, each with the normal anatomy displayed in the top and simulated atrophic anatomy in the bottom. [Color figure can be viewed in the online issue, which is available at wileyonlinelibrary.com.] vector as the morphological signature to guide the registration. In our method, we simultaneously estimate the transformation fields for all subjects in the population, and warp them towards a hidden common space. To efficiently solve the complicated optimization problem in groupwise registration, we hierarchically select the driving voxels to steer the groupwise registration. By taking advantage of the driving voxels (with distinctive features) automatically detected from all images, we develop a feature-based groupwise registration framework by alternatively establishing the correspondences on the driving voxels by soft assignment strategy and estimating dense transformation fields by TPS. Extensive experiments have been performed to compare the performance of our method with that of the congealing method and the pairwise HAMMER algorithm. All experimental results show that our method can achieve the best performance.

Our future work includes integration of more image features into our groupwise registration framework, to further improve its performance. Also, we will apply our method to more clinical datasets, to demonstrate its power in clinical applications.

TABLE II. Paired $t$-test results on simulated brain atrophy detection

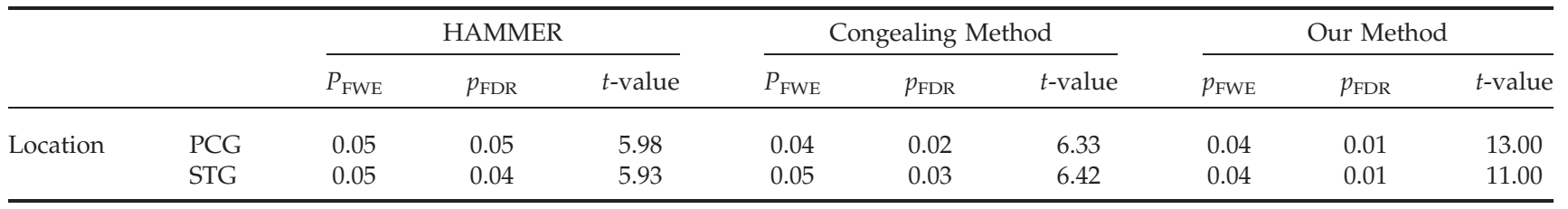

TABLE III. Computation times of congeal and our registration methods

\begin{tabular}{lcccc}
\hline Dataset & Subject number & Image size & Time by congealing (h) & Time by our method (h) \\
\hline Elderly brains & 18 & $256 \times 256 \times 124$ & 8.0 & 8.5 \\
NIREP & 16 & $256 \times 300 \times 256$ & 8.3 & 9.5 \\
LONI LPBA40 & 40 & $181 \times 271 \times 181$ & 19.4 & 19.2 \\
Simulated atrophy & 24 & $256 \times 256 \times 124$ & 10.6 & 11.4 \\
\hline
\end{tabular}




\section{REFERENCES}

Allassonnière S, Amit Y, Trouvé A (2007): Towards a coherent statistical framework for dense deformable template estimation. Journal of the Royal Statistical Society: Series B (Statistical Methodology) 69:3-29.

Avants B, Epstein CL, Grossman M, Gee JC (2008): Symmetric diffeomorphic image registration with cross-correlation: Evaluating automated labeling of elderly and neurodegenerative brain. Med Image Anal 12:26-41.

Balci SK, Golland P, Shenton M, Wells WM (2007): Free-form Bspline deformation model for groupwise Registration. Med Image Comput Comput Assist Interv (MICCAI) 10:23-30.

Beg MF, Miller MI, Trouvé A, Younes L (2005): Computing large deformation metric mappings via Geodesic flows of diffeomorphisms. Int J Comput Vis 61:139-157.

Besl P, McKay N (1992): A method for registration of 3-D Shapes. IEEE Trans Pattern Anal Mach Intell 14:239-256.

Bhatia KK, Hajnal JV, Puri BK, Edwards AD, Rueckert D (2004): Consistent groupwise non-rigid registration for atlas construction. IEEE Int Symp Biomed Imag: Macro Nano 1:908-911.

Blezek DJ, Miller JV (2007): Atlas stratification. Med Image Anal 11:443-457.

Bookstein FL (1989a): Principal warps: Thin-plate splines and the decomposition of deformations. Pattern Analysis and Machine Intelligence. IEEE Trans 11:567-585.

Bookstein FL (1989b): Principal warps: Thin-plate splines and the decomposition of deformations. IEEE Trans Pattern Anal Mach Intell 11:567-585.

Cachier P, Bardinet E, Dormont D, Pennec X, Ayache N (2003): Iconic feature based nonrigid registration: The PASHA algorithm. Comput Vis Image Underst 89:272-298.

Christensen G, Geng X, Kuhl J, Bruss J, Grabowski T, Pirwani I, Vannier M, Allen J, Damasio H (2006a): Introduction to the non-rigid image registration evaluation project (NIREP). Biomed Image Reg 4057:128-135.

Christensen GE (1999): Consistent linear-elastic transformations for image matching. Lect Notes Comput Sci 224-237.

Christensen GE, Johnson HJ (2001): Consistent image registration. IEEE Trans Med Imaging 20:568-582.

Christensen GE, Geng X, Kuhl JG, Bruss J, Grabowski TJ, Pirwani IA, Vannier MW, Allen JS, Damasio H (2006b): Introduction to the Non-rigid Image Registration Evaluation Project (NIREP) 3rd International Workshop on Biomedical Image Registration LNCS 4057:128-135.

Chui H, Rangarajan A (2003): A new point matching algorithm for non-rigid registration. Comput Vis Image Underst 89:114-141.

Cootes TF, Marsland S, Twining CJ, Smith K, Taylor CJ (2004): Groupwise diffeomorphic non-rigid registration for automatic model building. 8th European Conference on Computer Vision (ECCV) 3024:316-327.

Crum WR, Hartkens T, Hill DLG (2004): Non-rigid image registration: Theory and practice. Br J Radiol 77 (Suppl 2):S140-S153.

Davatzikos C, Genc A, Xu D, Resnick SM (2001): Voxel-based morphometry using the RAVENS maps: Methods and validation using simulated longitudinal atrophy. NeuroImage 14: 1361-1369.

Davis BC, Fletcher PT, Bullitt E, Joshi S (2007) Population shape regression from random design data. IEEE 11th International Conference on Computer Vision ICCV.2007.4408977:1-7.

Fletcher PT, Venkatasubramanian S, Joshi S (2009): The geometric median on Riemannian manifolds with application to robust atlas estimation. NeuroImage 45(1, Suppl 1):S143-S152.
Geng X, Christensen GE, Gu H, Ross TJ, Yang Y (2009): Implicit reference-based group-wise image registration and its application to structural and functional MRI. NeuroImage 47: 1341-1351.

Jaccard P (1912): The distribution of the flora in the alpine zone. New Phytologist 11:37-50.

Joshi S, Davis B, Jomier M, Gerig G (2004): Unbiased diffeomorphic atlas construction for computational anatomy. Neuroimage 23 (Suppl 1):S151-S160.

Learned-Miller EG (2006): Data driven image models through continuous joint alignment. Pattern Analysis and Machine Intelligence. IEEE Trans 28:236-250.

Liao S, Chung A (2008): Non-rigid image registration with S $\alpha$ S Filters. Med Image Comput Comput Assist Interv (MICCAI) 5242:888-896.

Lo CH, Don HS (1989): 3-D Moment forms: Their construction and application to object identification and positioning. IEEE Trans Pattern Anal Mach Intell 11:1053-1064.

Maintz JBA, Viergever MA (1998): A survey of medical image registration. Med Image Anal 2:1-36.

Marsland S, Twining CJ, Taylor CJ (2008): A minimum description length objective function for groupwise non-rigid image registration. Image Vis Comput 26:333-346.

Miller EG, Matsakis NE, Viola PA (2000): Learning from one example through shared densities on transforms. Proc IEEE Conf Comput Vis Pattern Recogn 1:1464

Resnick SM, Goldszal A, Davatzikos C, Golski S, Kraut MA, Metter EJ, Bryan RN, Zonderman AB (2000): One-year age changes in MRI brain volumes in older adults. Cerebral Cortex 10:464-472.

Rueckert D, Sonoda LI, Hayes C, Hill DLG, Leach MO, Hawkes DJ (1999): Non-rigid registration using free-form deformations: Application to breast MR images. IEEE Trans Med Imaging 18:712-721.

Sabuncu MR, Balci SK, Shenton ME, Golland P (2009): Imagedriven population analysis through mixture modeling. Med Imaging IEEE Trans 28:1473-1487.

Seghers D, Agostino DE, Maes F, Vandermeulen D, Suetens P (2004): Construction of a brain template from MR images using state-of-the-art registration and segmentation techniques. Med Image Comput Comput Assist Interv (MICCAI) 696-703.

Shattuck DW, Mirza M, Adisetiyo V, Hojatkashani C, Salamon G, Narr KL, Poldrack RA, Bilder RM, Toga AW (2008): Construction of a 3D probabilistic atlas of human cortical structures. Neuroimage 39:1064-1080.

Shen D (2007): Image registration by local histogram matching. Pattern Recogn 40:1161-1172.

Shen D (2009): Fast image registration by hierarchical soft correspondence detection. Pattern Recogn 42:954-961.

Shen D, Davatzikos C (2002): HAMMER: Hierarchical attribute matching mechanism for elastic registration. IEEE Trans Med Imag 21:1421-1439.

Shen D, Davatzikos C (2003): Very high resolution morphometry using mass-preserving deformations and HAMMER elastic registration. NeuroImage 18:28-41.

Studholme C, Cardenas V (2004): A template free approach to volumetric spatial normalization of brain anatomy. Pattern Recogn Lett 25:1191-1202.

Thirion JP (1998): Image matching as a diffusion process: An analogy with Maxwell's demons. Med Image Anal 2:243-260.

Vercauteren T, Pennec X, Perchant A, Ayache N (2009a): Diffeomorphic demons: Efficient non-parametric image registration. Neuroimage 45(1, Suppl 1):S61-S72. 
Vercauteren T, Pennec X, Perchant A, Ayache N (2009b): Diffeomorphic demons: Efficient non-parametric image registration. Neuroimage 45:S61-S72.

Wang Q, Wu G, Yap P-T, Shen D (2010): Attribute vector guided groupwise registration. NeuroImage 50:1485-1496.

Wu G, Qi F, Shen D (2006a): A general learning framework for nonrigid image registration; August 17-18 2006; Shanghai, China.

Wu G, Qi F, Shen D (2006b): Learning-based deformable registration of MR brain images. IEEE Trans Med Imaging 25: 1145-1157.

Wu G, Qi F, Shen D (2007): Learning Best Features and Deformation Statistics for Hierarchical Registration of MR Brain Images. Inf Proc Med Ima, The Netherlands. p 160-171.
Wu G, Yap P-T, Kim M, Shen D (2010): TPS-HAMMER: Improving HAMMER registration algorithm by soft correspondence matching and thin-plate splines based deformation interpolation. NeuroImage 49:2225-2233.

Xue Z, Shen D, Karacali B, Stern J, Rottenberg D, Davatzikos C (2006): Simulating deformations of MR brain images for validation of atlas-based segmentation and registration algorithms. NeuroImage 33:855-866.

Yap P, Wu G, Zhu H, Lin W, Shen D (2009) Fast tensor image morphing for elastic registration. Medical Image Computing and Computer-Assisted Intervention - MICCAI LNCS 5761:721-729

Zitová B, Flusser J (2003): Image registration methods: A survey. Image Vision Comp 21:977-1000. 